\title{
Lineage fate of ductular reactions in liver injury and carcinogenesis
}

\author{
Simone Jörs, ${ }^{1}$ Petia Jeliazkova, ${ }^{1}$ Marc Ringelhan, ${ }^{1,2,3}$ Julian Thalhammer, ${ }^{1}$ Stephanie Dürl, ${ }^{1}$ Jorge Ferrer, ${ }^{4}$ Maike Sander, ${ }^{5}$ \\ Mathias Heikenwalder, ${ }^{2}$ Roland M. Schmid, ${ }^{1,6}$ Jens T. Siveke, ${ }^{1,6}$ and Fabian Geisler ${ }^{1}$ \\ ²nd Department of Internal Medicine, Klinikum rechts der Isar, Technische Universität München, Munich, Germany. ${ }^{2}$ Institute for Virology, Technische Universität München/Helmholtz Zentrum München, \\ Munich, Germany. ${ }^{3}$ German Centre for Infection Research (DZIF), Munich Partner Site, Munich, Germany. ${ }^{4}$ Department of Medicine, Imperial College London, London, United Kingdom. \\ ${ }^{5}$ Departments of Pediatrics and Cellular and Molecular Medicine, Pediatric Diabetes Research Center, University of California, San Diego, La Jolla, California, USA. \\ ${ }^{6}$ Cerman Cancer Consortium (DKTK) and German Cancer Research Center (DKFZ), Heidelberg, Germany
}

\begin{abstract}
Ductular reactions (DRs) are observed in virtually all forms of human liver disease; however, the histogenesis and function of DRs in liver injury are not entirely understood. It is widely believed that DRs contain bipotential liver progenitor cells (LPCs) that serve as an emergency cell pool to regenerate both cholangiocytes and hepatocytes and may eventually give rise to hepatocellular carcinoma (HCC). Here, we used a murine model that allows highly efficient and specific lineage labeling of the biliary compartment to analyze the histogenesis of DRs and their potential contribution to liver regeneration and carcinogenesis. In multiple experimental and genetic liver injury models, biliary cells were the predominant precursors of DRs but lacked substantial capacity to produce new hepatocytes, even when liver injuries were prolonged up to 12 months. Genetic modulation of NOTCH and/or WNT/ $\beta$-catenin signaling within lineage-tagged DRs impaired DR expansion but failed to redirect DRs from biliary differentiation toward the hepatocyte lineage. Further, lineage-labeled DRs did not produce tumors in genetic and chemical HCC mouse models. In summary, we found no evidence in our system to support mouse biliary-derived DRs as an LPC pool to replenish hepatocytes in a quantitatively relevant way in injury or evidence that DRs give rise to HCCs.
\end{abstract}

\section{Introduction}

Ductular reactions (DRs) comprise cellular reactions of ductular phenotype at the portal-parenchymal interface and can be observed in response to acute or chronic injury in virtually all forms of human liver disease $(1,2)$. There is a marked diversity in DRs with respect to morphological appearance as well as their putative cellular origin, possibly reflecting the mode of injury. It is widely accepted that, in most cases, DRs arise from the biliary compartment, either from mature bile ducts or their outermost terminals, the canals of Hering $(1,3,4)$. Hence, ductular reactive cells (DRCs) arise periportally and express biliary proteins, such as CK19, EpCAM, CD133, SOX9, or A6 (rodents). However, hepatocytes may also give rise to DRCs by undergoing biliary metaplasia in response to injury (5-8) and have recently even been proposed to be the main cellular source for DRs in distinct injury models (9).

The explicit functional relevance of DRs in liver disease is not entirely understood. Data derived from histomorphology and in vitro studies have long suggested that DRs may contain progenitors that normally reside in a quiescent state in the canals of Hering of the normal liver and have led to the frequent synonymous use of the terms DRCs, liver progenitor cells (LPCs), or adult hepatic stem cells. It is widely believed that such putative

Authorship note: Simone Jörs and Petia Jeliazkova contributed equally to this work Conflict of interest: The authors have declared that no conflict of interest exists. Submitted: August 17, 2014; Accepted: March 20, 2015.

Reference information: / Clin Invest. 2015;125(6):2445-2457. doi:10.1172/JCI78585. progenitors serve as an adult emergency compartment capable of producing both cholangiocytes and hepatocytes in case acute or chronic liver injury compromises self-duplication of these mature epithelial cells. While the biliary fate is considered the "default setting" of DRCs determined by juxtacrine NOTCH activation, paracrine activation of $\beta$-catenin by WNT proteins may alter the fate of biliary-derived DRCs such that they become hepatocytes (10-12). In line with this concept of DRCs physiologically acting as bipotential progenitors, cells isolated from DRs can self-renew, give rise to biliary cells and hepatocytes in vitro, and regenerate hepatocytes with variable success when transplanted into $\mathrm{Fah}^{-1-}$ mice, a mouse model of hereditary tyrosinemia type 1 (13-16). Yet, recent attempts to address the capacity of DRCs to act as physiological progenitors to regenerate hepatocytes after short-term liver injury using genetic lineage-tracing methods have revealed conflicting results $(14,17-23)$. Beyond liver repair, LPCs have also come into focus as a likely candidate cellular compartment susceptive to malignant transformation and that can give rise to hepatocellular carcinoma (HCC) or cholangiocarcinoma $(24,25)$; however, evidence from compartment-specific lineage-tracing in vivo models that supports the view that HCCs originate from an adult LPC compartment does not exist.

Here, by using a highly specific lineage-tracing system for the adult biliary compartment that allows efficient long-term tracking of DRs in a broad range of different liver injury models, we provide a comprehensive analysis to define the histogenesis of DRs and their prospective cellular fate in liver regeneration and 
A
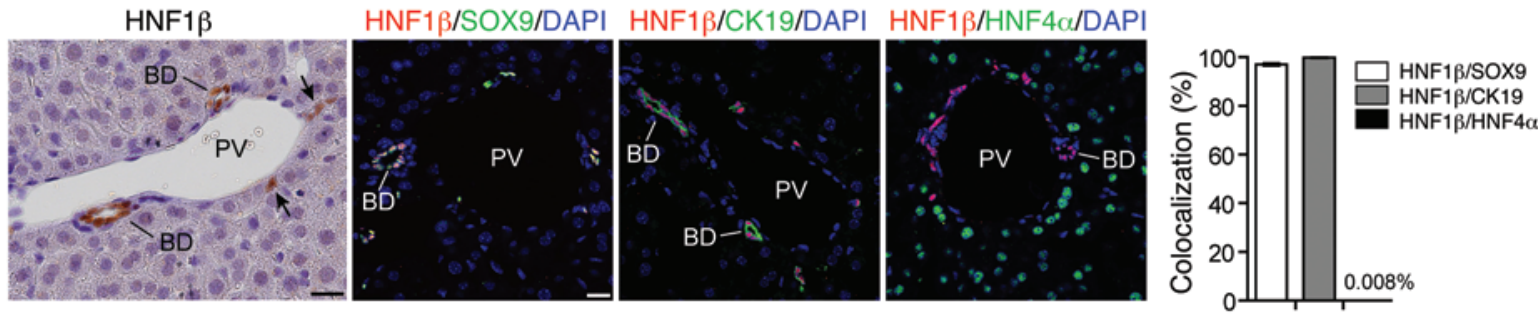

B
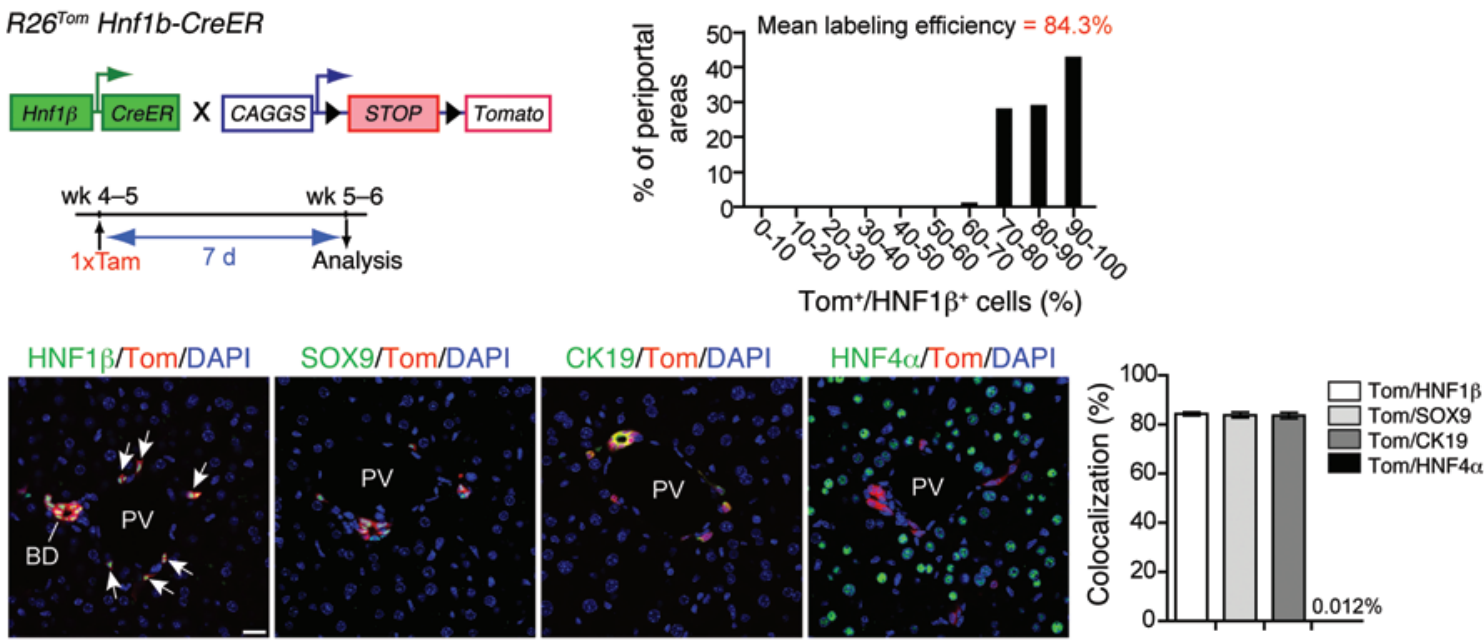

Figure 1. Efficient and specific inducible lineage labeling of the biliary compartment in R26 ${ }^{\text {Tom }}$ Hnf1b-CreER animals. (A) In the adult mouse, HNF1 $\beta$ was specifically expressed in bile ducts and biliary ductules/canals of Hering (arrows). HNF1 $\beta^{+}$cells were identical with SOX9+ and CK19+ cells, as assessed by co-IF (HNF1 $\beta^{+} / \mathrm{SOX9}^{+}: 97.04 \% \pm 0.52 \%, n=3$; HNF1 $\beta^{+} / \mathrm{CK}_{19}{ }^{+}$: $99.89 \% \pm 0.10 \%, n=5$ ), while HNF1 $\beta$ expression in HNF4 $\alpha^{+}$hepatocytes was virtually absent $(0.008 \% \pm 0.004 \%, n=8)$. (B) Cre-induced expression of the fluorescent dye tdTom was determined in 5- to 6-week-old R26 Tom Hnf1b-CreER reporter mice 7 days after a single tamoxifen injection. tdTom expression was restricted to HNF1 $\beta^{+} / \mathrm{SOXg}^{+} / \mathrm{CK} 19^{+}$bile ducts and periportal ductules $(n=5)$, whereas labeling of $\mathrm{HNF} 4 \alpha^{+}$hepatocytes was a rare event $(0.012 \% \pm 0.003 \%, n=10)$. All periportal areas revealed a high tdTom labeling efficacy of the HNF1 $\beta^{+}$ compartment ( $>30$ portal tracts per mouse were quantified at $\times 600$ magnification from 3 discontinuous sections; mean labeling efficiency: $84.3 \% \pm 0.6 \%$; $n=5$ animals). BD, bile duct; PV, portal vein; Tam, tamoxifen. Scale bar: $20 \mu \mathrm{m}$.

carcinogenesis and close important knowledge gaps within this controversial field.

\section{Results}

DRs arise from biliary $H N F 1 \beta^{+}$cells in a broad range of liver injury models. In the postnatal liver, HNF1 $\beta$ is expressed in bile ducts and periportal ductules of the canals of Hering. $\mathrm{HNF} 1 \beta^{+}$cells costained for the biliary markers CK19 and SOX9 but showed no overlapping staining with the hepatocyte marker HNF $4 \alpha$ (Figure 1A and ref. 7). These observations suggest that HNF1 $\beta$ expression discriminates between cells of the biliary and hepatocyte compartment in the postnatal liver. We also found intense HNF1 $\beta$ staining of DRCs after different liver injuries, prompting us to trace the fate of postnatal biliary HNF1 $\beta^{+}$cells as putative precursors of DRs in liver injury. We have previously shown that tamoxifen-inducible expression of the red fluorescent dye tdTomato (tdTom) (26) in adult $R 26^{\text {Tom }} \mathrm{Hnf1b}$-CreER animals allows highly specific and efficient lineage labeling of the HNF1 $\beta^{+}$biliary compartment (7). Here, tdTom labeling efficiency of the biliary compartment was consistently approximately $84 \%$, as assessed by costaining for HNF1 1 , CK19, or SOX9 1 week after a single tamoxifen injection (i.p., $100 \mu \mathrm{g} / \mathrm{g} \mathrm{BW}$ ) in 4- to 5-week-old R26 ${ }^{\text {Tom }} \mathrm{Hnflb}$-CreER animals, while tdTom expression in hepatocytes was an extremely rare event, found in $<1: 8,000 \mathrm{HNF} 4 \alpha^{+}$hepatocytes (Figure 1B).
To assess whether DRs are the progeny of the HNF1 $\beta^{+}$ductal compartment, HNF1 $\beta$ lineage-labeled $R 26^{\text {Tom }}$ Hnflb-CreER animals were subjected to 2 to 3 weeks of various liver injury models known to elicit a DR after predominantly biliary (bile duct ligation [BDL]; methylene dianiline [MDA] treatment), mixed hepatocellular-cholestatic (3,5-diethoxycarbonyl-1,4-dihydrocollidine [DDC] diet), or hepatocellular injury (choline-deficient ethionine-supplemented [CDE] diet) models. In addition to these exogenous models, we used a genetic mouse model of chronic hepatocellular-cholestatic injury ( $M d r 2^{-/-} R 26^{\text {Tom }} \mathrm{Hnf1b}$-CreER animals; tamoxifen treatment at P21) analyzed at P60 (Figure 2A). Irrespective of the injury model used, we found up to $85 \% \mathrm{CK} 19^{+} \mathrm{DRCs}$ costaining for tdTom (Figure 2, B and C), indicating that the vast majority of DRCs arise from the ductal HNF1 $\beta^{+}$compartment. Notably, tdTom/HNF $4 \alpha$ costaining did not reveal significant neogenesis of hepatocytes from DRCs in our injury models, with the exception of the 2-week CDE model, in which a very small fraction $(0.04 \% \pm 0.019 \%)$ of $\operatorname{tdTom}^{+}$/ $\mathrm{HNF} 4 \alpha^{+}$hepatocytes was observed (Figure 2, B and C).

The DDC and CDE models are considered prototypical mouse "oval cell models" that produce bipotential LPCs; however, their potential and quantitative contribution to reparative hepatocyte neogenesis remains controversial (10,14,17-23). Neither introducing a 2-week recovery period (DDC diet for 2 weeks plus 2 weeks recovery) nor prolonging DDC feeding (DDC diet for 4 weeks) 
A R26 $6^{\text {Tom }} \mathrm{Hnf1b}$-CreER

DDC 2 wks: $\underset{\text { Tam } 5}{\text { wk } 5} \quad$ wk 8

BDL 2 wks:

\begin{tabular}{ccc} 
wk 7 & wk 8 & wk 10 \\
\hdashline$\uparrow$ & $\uparrow$ & $\vdots$ \\
Tam & BDL & Analysis
\end{tabular}

MDA 3 wks:

\begin{tabular}{cccc} 
wk 5 & wk 6 & wk 7 & wk 9 \\
\hline$\uparrow$ & $\uparrow \uparrow \uparrow$ & $\downarrow$ \\
Tam & MDA $3 x$ & Analysis
\end{tabular}

CDE 2 wks:

$\underset{\text { Tam }}{\text { wk } 4}$ wk $5 \quad$ wk 7

Mdr2 ${ }^{-/}$R26 $6^{\text {Tom }}$ Hnf1b-CreER

$M d r 2^{-1-8}$ wks: wk 3-4

$\overbrace{\text { Tam }}^{+1}$

B

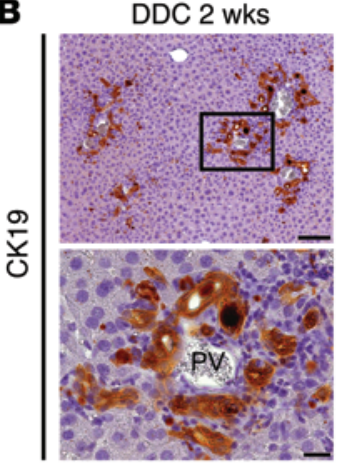

BDL 2 wks
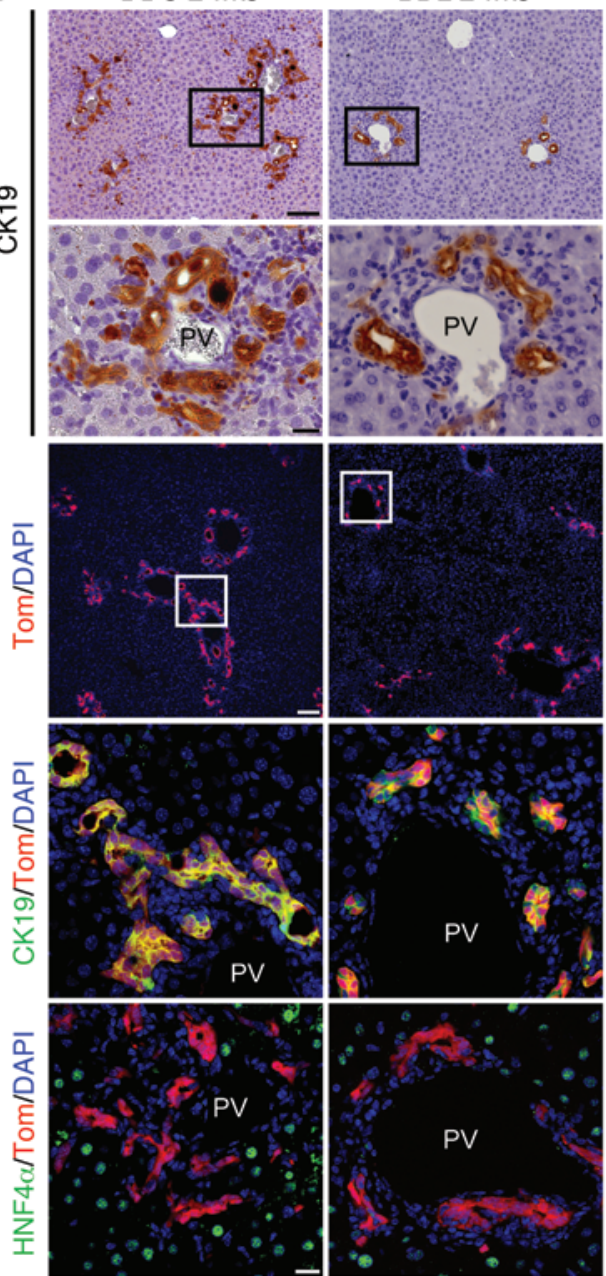

C

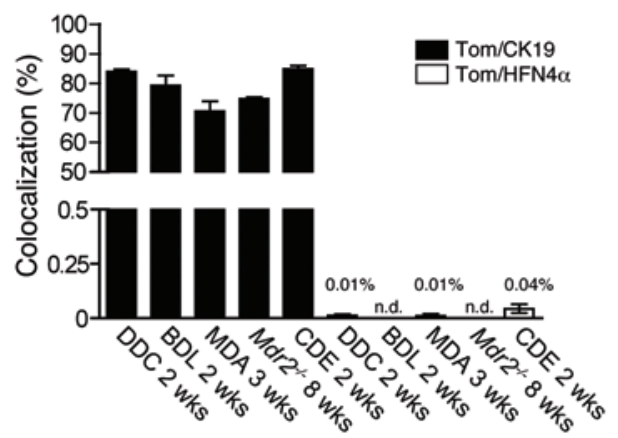

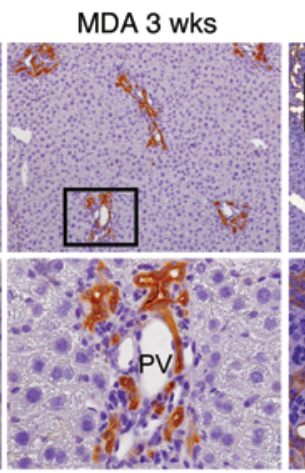

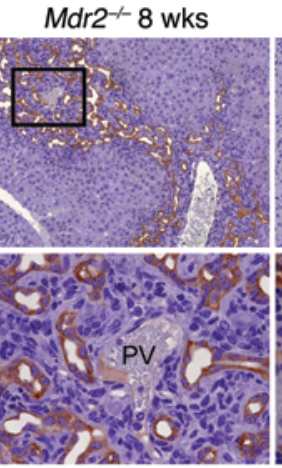

CDE 2 wks
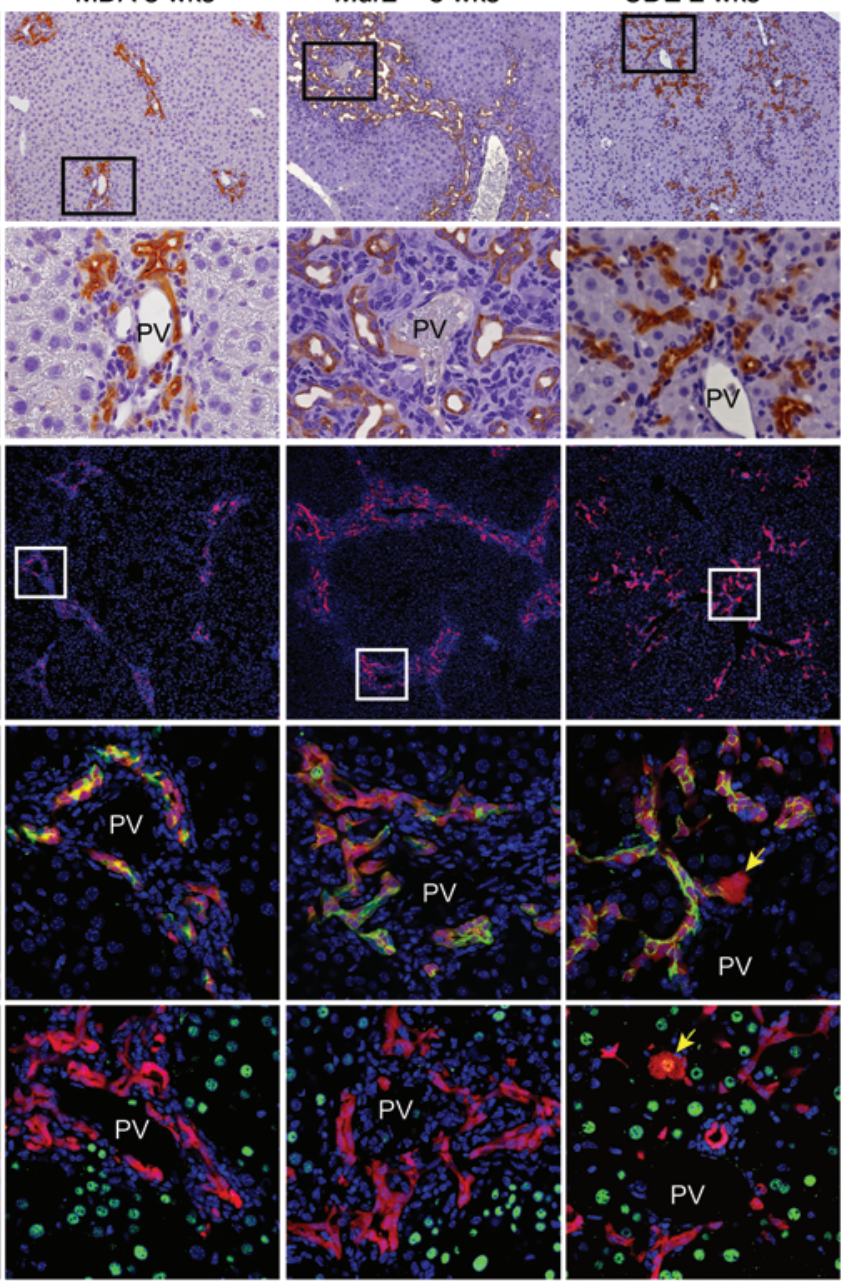

Figure 2. DRs arise from the biliary HNF1 ${ }^{+}$compartment in a broad range of liver injury models. (A) Seven days after tamoxifen injection, $R 26^{\text {Tom }}$ Hnf1b-CreER mice were subjected to 2 to 3 weeks of various short-term liver injury models (DDC diet, BDL, MDA, CDE diet). For fate mapping of DRs in MDR2-deficient mice, Mdr2 ${ }^{-1-} R 26^{\text {Tom }} \mathrm{Hnf1b}$-CreER animals received tamoxifen at week 3 to 4 and were analyzed at week 8. (B) In all models, characteristic DRs were assessed by IHC for CK19. Regardless of the mode of injury co-IF analysis revealed tdTom expression in most CK19+ cells, while expression of tdTom in HNF4 $\alpha^{+}$hepatocytes was consistently only observed in the CDE model (yellow arrows). (C) Colocalization (mean value \pm SEM) of tdTom ${ }^{+} / \mathrm{CK}_{19}{ }^{+}$ and tdTom ${ }^{+} / \mathrm{HNF} \alpha^{+}$cells expressed as percentage colocalization (DDC diet, $n=9 ; \mathrm{BDL}, n=4$; MDA, $n=4 ; \mathrm{Mdr2}^{-/-}, n=4 ; \mathrm{CDE}$ diet, $n=5$ ). n.d., not detected. Scale bar: $100 \mu \mathrm{m}$ (first and third rows); $20 \mu \mathrm{m}$ (second, fourth, and fifth rows). 
A

R26 ${ }^{\text {Tom }}$ Hnf1b-CreER

Mdr2 $^{--}$R26 ${ }^{\text {Tom }}$ Hnf1b-CreER

R26 ${ }^{\text {Tom }}$ Hnf1b-CreER
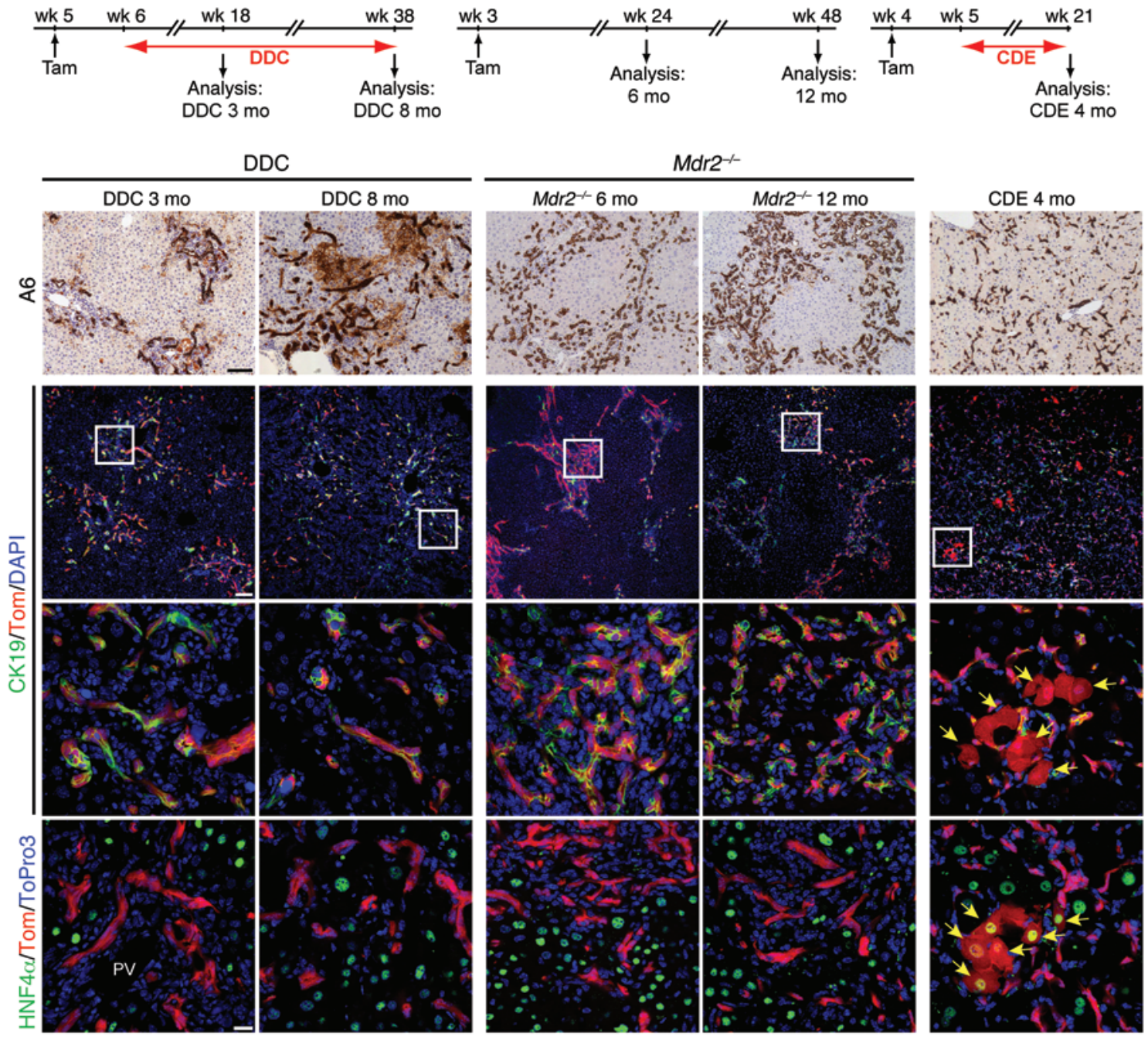

B
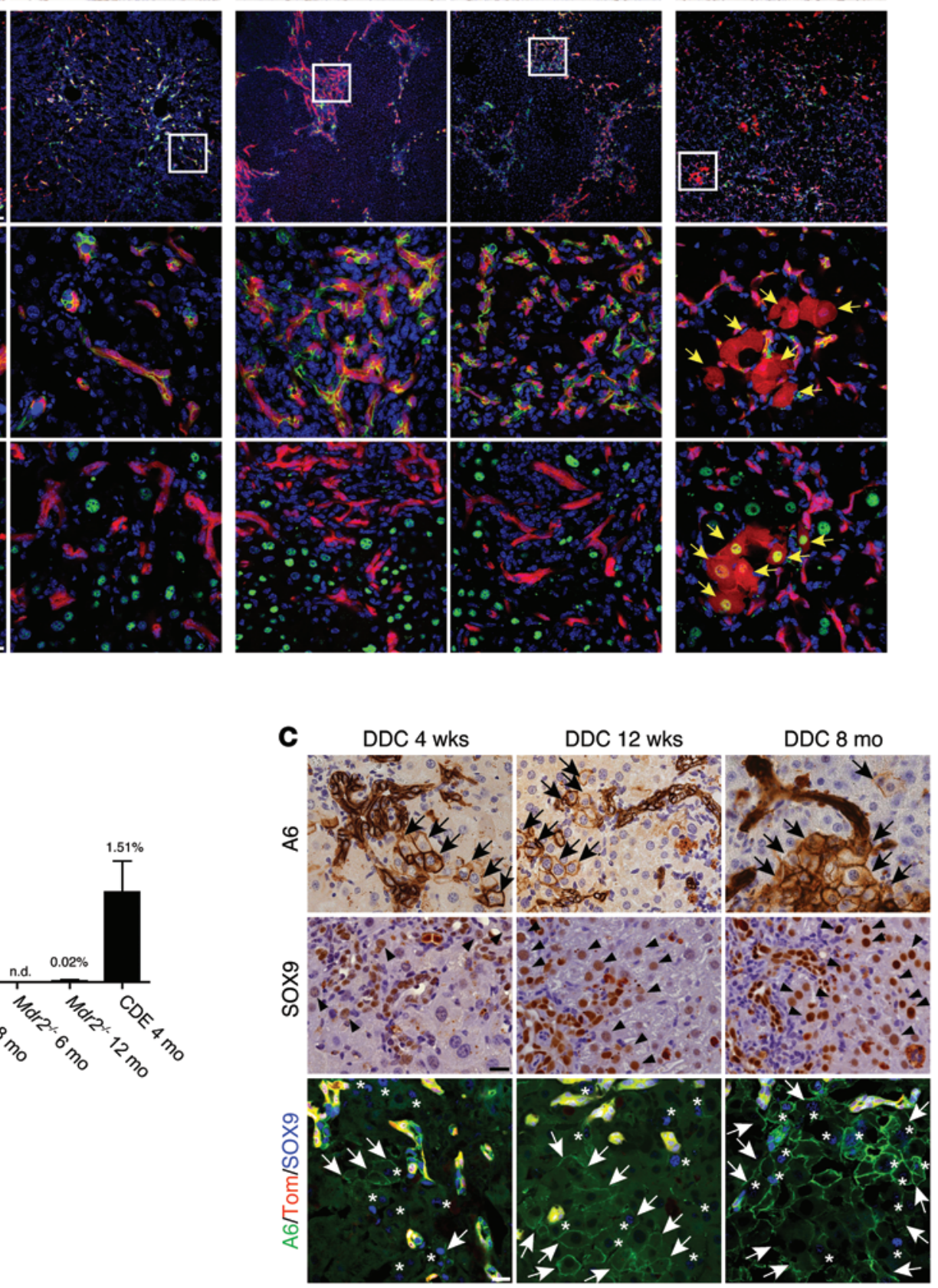
Figure 3. Minimal contribution of DRCs to hepatocyte neogenesis after long-term liver injury. (A) We traced the fate of lineage-labeled DRs in $R 26^{\text {Tom }} \mathrm{Hnf} 1 \mathrm{~b}$-CreER animals after prolonged DDC diet (up to 8 months) or CDE diet (4 months) as well as lineage-labeled DRs in $\mathrm{Mdr}^{-1-} \mathrm{R} 26^{\text {Tom }}$ Hnf1b-CreER animals (up to 12 months). Despite dramatic expansions of $\mathrm{A6}^{+}(\mathrm{IHC})$ or tdTom ${ }^{+} / \mathrm{CK} 19^{+}$(IF) DRs in all long-term injury models, only mice fed CDE diet for 4 months displayed a small fraction of $\mathrm{HNF} \alpha^{+}$hepatocytes coexpressing tdTom (IF, yellow arrows). Representative pictures are shown of $n=4$ to 6 animals analyzed per group. (B) Proportion of $H N F 4 \alpha^{+}$ hepatocytes that coexpress tdTom. tdTom ${ }^{+} / \mathrm{HNF} \alpha^{+}$hepatocytes (mean value \pm SEM) were quantified from $n=4$ to 6 animals per group. (C) In the DDC model, IHC analysis for A6 and SOX9 shows hepatocytes and cells of intermediate morphology that express biliary markers (arrows and arrowheads in IHC panels), with increasing time of injury surrounding typical $\mathrm{A6}^{+}$ DRs. Co-IF analysis for tdTom, A6, and SOX9 revealed no coexpression of tdTom in $\mathrm{A6}^{+}$or $\mathrm{SOX9^{+ }}$ hepatobiliary cells (arrows and asterisks), indicating their hepatocyte origin (for quantification, refer to Supplemental Figure 5). Scale bar: $100 \mu \mathrm{m}$ (top 2 rows, $\mathbf{A}$ ); $20 \mu \mathrm{m}$ (bottom 2 rows, $\mathbf{A}$, and $\mathbf{C}$ ).

produced a relevant number of tdTom $/ \mathrm{HNF}^{+} \alpha^{+}$hepatocytes (Supplemental Figure 1A; supplemental material available online with this article; doi:10.1172/JCI78585DS1). In contrast, prolonging $\mathrm{CDE}$ diet produced $0.13 \% \pm 0.05 \% \mathrm{tdTom}^{+}$hepatocytes $(\mathrm{CDE}$ diet for 3 weeks) and $2.12 \% \pm 0.48 \% \mathrm{HNF}^{2} \alpha^{+} / \mathrm{tdTom}^{+}$hepatocytes after introducing a recovery phase (CDE diet for 3 weeks plus 2 weeks recovery) (Supplemental Figure 1B). Although 2/3 partial hepatectomy $(\mathrm{PH})$ is considered the prototypical model for liver mass regeneration by self-renewal of hepatocytes, recent data proposed that DRCs contribute to hepatocyte neogenesis in this model $(17,18)$. However, even after extended $80 \%$ PH $(27)$, we did not find hepatocyte regeneration from biliary progenitors (Supplemental Figure 1C, left). Further, $2 / 3 \mathrm{PH}$ preceded by pretreatment of mice with retrorsine, used to inhibit hepatocyte replication in mice (28), elicited a moderate td Tom ${ }^{+}$DR that did not significantly contribute to hepatocyte neogenesis (Supplemental Figure 1C, right). Notably, liver mass recovery in this model was normal but was mainly achieved by hepatocyte hypertrophy, as the number of hepatocytes per high-power field was greatly reduced after $\mathrm{PH}$ when compared with that of controls $(83.5 \pm 9.4$ hepatocytes vs. $196.7 \pm 4.8$ hepatocytes per $\times 200$ high-power field; $n=4$ and $n=3$ ).

We found generation of hepatocytes from DRCs to be a rare event after short-term injury and restricted to the CDE model. Thus, our results suggest that DRCs derived from the HNF1 $\beta^{+}$compartment rarely act as hepatocyte progenitors. In addition, conflicting data exist with regard to the contribution of such progenitors to hepatocyte turnover during normal homeostasis $(4,17-19$, 29). When $R 26^{\text {Tom }} H n f 1 b$-CreER animals were analyzed 6 months after tamoxifen treatment, we did not detect duct-derived hepatocytes, as assessed by tdTom/HNF4 $\alpha$ costaining (Supplemental Figure 2, A and B). We also used a Sox9-CreER mouse strain to trace the fate of $\mathrm{HNF} 1 \beta^{+} / \mathrm{SOX}^{+}$cells $(14,30)$. Like HNF $1 \beta$, SOX9 expression is restricted to intrahepatic bile ducts and ductules showing no overlap with the $\mathrm{HNF} 4 \alpha^{+}$hepatocyte compartment in postnatal mice $(4,7,31)$. However, tamoxifen injection into $R 26^{\text {Tom }}$ Sox9-CreER animals resulted not only in effective tdTom labeling of biliary cells but also in consistent labeling of 1 to 2 layers of periportal zone 1 hepatocytes (Supplemental Figure 2, A and B). As suggested previously, this "ectopic" labeling of periportal hepa- tocytes in the Sox9-CreER mouse strain most likely results from tamoxifen-induced activation of the Cre-driving Sox 9 promoter in normally SOX9- hepatocytes $(4,20)$. Although this significant overlap of cellular compartments prevents the use of this mouse strain to trace the fate of biliary-derived $\mathrm{SOX9}^{+}$ductal cells as they differentiate to hepatocytes, it allows lineage tracing of zone 1 hepatocytes to reappraise the "streaming liver" hypothesis coined by Zajicek 30 years ago (32). However, the fraction of tdTom ${ }^{+}$ hepatocytes in tamoxifen-treated $R 26^{\text {Tom }}$ Sox9-CreER livers did not change significantly after 6 months, and tdTom ${ }^{+} / \mathrm{HNF} \alpha^{+}$ hepatocytes did not leave zone 1 (Supplemental Figure 2, A and B). From these two different lineage-tracing models, we conclude that during normal liver homeostasis there is no "streaming" of hepatocytes, which are constantly renewed either by $\mathrm{SOX}^{+} /$ HNF1 $\beta^{+}$ductal progenitors or by periportal zone 1 hepatocytes.

$D R C s$ do not significantly contribute to hepatocyte regeneration after long-term injury. After demonstrating that DRs do not significantly contribute to hepatocyte regeneration after short-term injury, we sought to characterize their regenerative capacity in long-term injury, a time period when progenitor-derived hepatocytes are speculated to accumulate. For this, we traced tdTom lineage-labeled DRCs after DDC or CDE long-term treatment or in mice lacking the MDR2 transporter. In contrast to livers with short-term DDC treatment, livers treated with DDC diet for 3 and 8 months displayed increasingly more parenchymal damage, accompanied by accumulative DRs that adapted a more stringlike morphology radiating into zone 2 and 3 (Figure 3A). However, even after 8 months of DDC treatment, we did not detect a relevant number of tdTom ${ }^{+}$hepatocytes (Figure 3B). Likewise, livers of $M d r 2^{-/} R 26^{\text {Tom }} \mathrm{Hnflb}$-CreER animals displayed large areas of tdTom $^{+} / \mathrm{CK}_{19}{ }^{+}$DRs after 6 and 12 months but lacked a significant number of $\mathrm{tdTom}^{+} / \mathrm{HNF}_{4} \alpha^{+}$hepatocytes (Figure 3, A and B). The most intense DR expansion was observed after 4 months of $\mathrm{CDE}$ treatment, the outermost tolerable treatment duration (CDE feeding had to be discontinued prematurely in 4 of 10 animals according to ethical guidelines). We found that the entire liver parenchyma (zones 1-3) was infiltrated by $\mathrm{tdTom}^{+} / \mathrm{CK} 9^{+} /$ $\mathrm{A}^{+}$DRCs. Livers were typically small, and the number of hepatocytes per high-power field was greatly reduced $(92.0 \pm 5.6$ hepatocytes vs. $237.7 \pm 29.9$ hepatocytes per $\times 200$ high-power field in age-matched controls) due to hepatocyte hypertrophy, indicating insufficient hepatocyte proliferation. Nevertheless, even in this severe long-term model with extensive parenchymal damage and high mortality, we did not detect a substantial number of td $\mathrm{Tom}^{+}$/ HNF $4 \alpha^{+}$hepatocytes $(1.51 \% \pm 0.53 \%)$ derived from the putative LPC compartment (Figure 3, A and B).

Notably, hepatocyte Ki67 labeling indices of up to 10\% in shortand long-term injury were observed in DDC-treated $R 26^{\text {Tom }} \mathrm{Hnflb}$ CreER or Mdr2 ${ }^{--}$R2 $^{\text {Tom }}$ Hnflb-CreER animals (summarized in Supplemental Figure 3A), indicating that hepatocyte replication is effective for hepatocyte repair in these models. In contrast, taking into account the extensive parenchymal injury observed in CDEtreated animals, Ki67 labeling of tdTom ${ }^{-}$hepatocytes was extremely low in the short-term model (CDE diet for 3 weeks: $0.21 \% \pm 0.13 \%$ ) and in the long-term model (CDE diet for 4 months: $1.86 \% \pm 0.39 \%$; Supplemental Figure 3B). The Ki67 index of tdTom ${ }^{+}$hepatocytes compared with that of tdTom ${ }^{-}$hepatocytes was higher in the CDE 
A R26 $6^{\mathrm{Tom}}$ Hnf1b-CreER

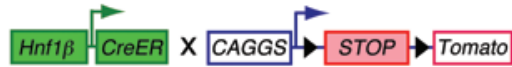

R26 ${ }^{\text {Tom }}$ Rbpj ${ }^{\text {fHI }}$ Hnf1b-CreER

Hnf1s $\overrightarrow{\text { CreER }} \times$ CAGGS
R26 ${ }^{\text {Tom }}$ Catnb $^{\Delta e x 3}$ Hnf1b-CreER

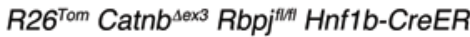

HNF1B CreER $x$ CAGGS

$\underset{\text { Tam }}{\text { wk } 5}$

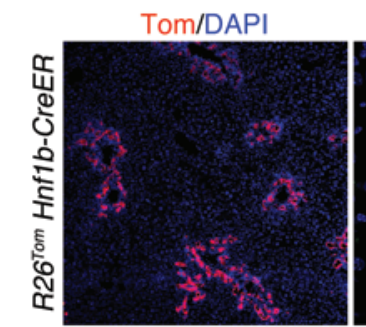

CK19/Tom/DAPI
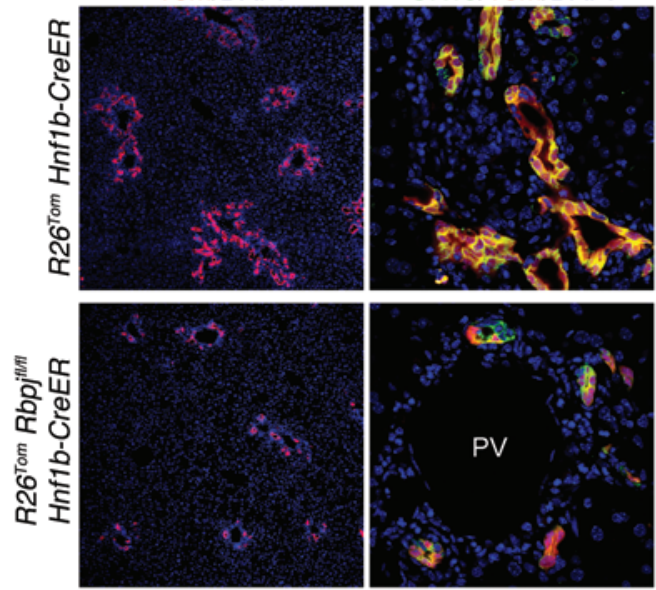

Ki67/Tom/ToPro3

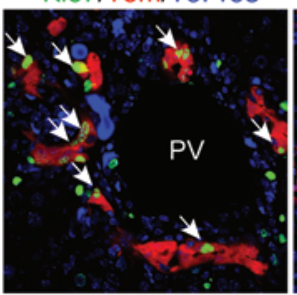

acTub/Tom/DAPI

HNF4 $\alpha$ Tom/ToPro3
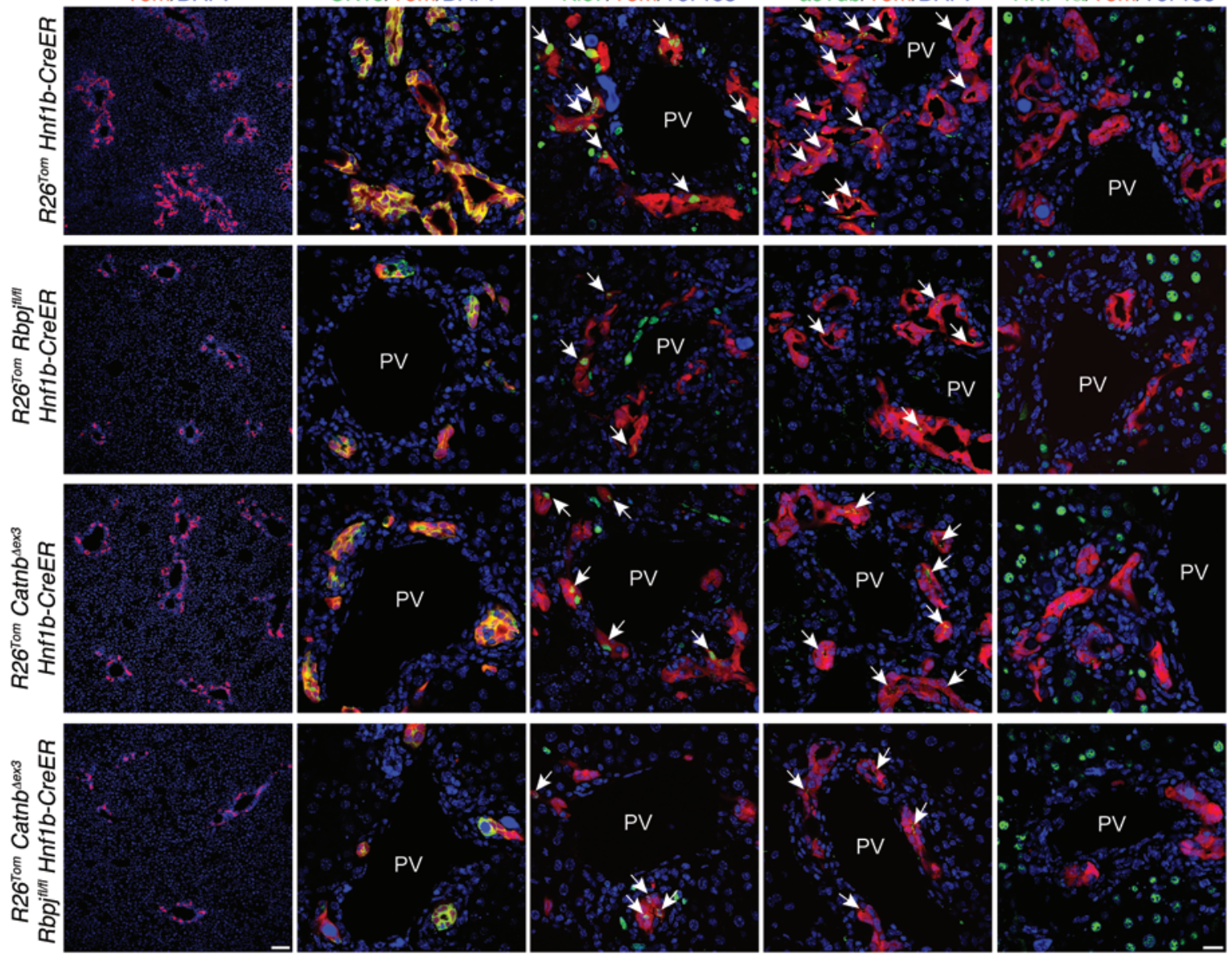

B
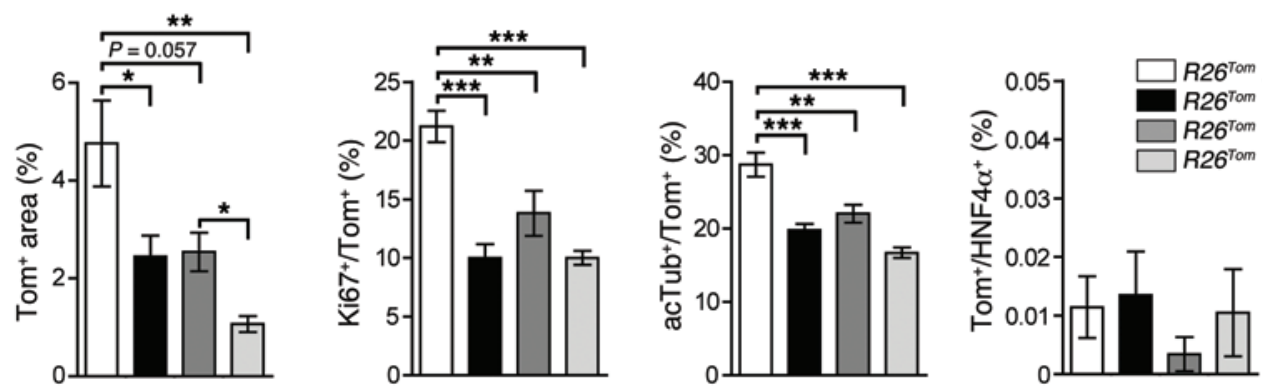

Figure 4. DDC-induced DRs do not escape their biliary fate after genetic ablation of NOTCH and/or activation of WNT/ $\beta$-catenin signaling. (A) For HNF1 $\beta$

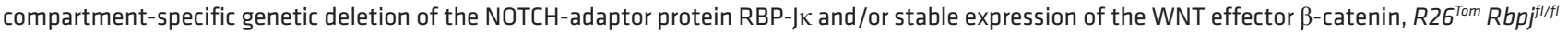

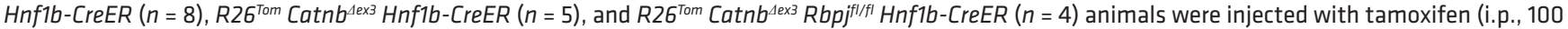
$\mu \mathrm{g} / \mathrm{g}$ BW at week 5) and subjected to 2 weeks of DDC diet (week 6-8). Equally treated R26 Tom $H n f 1 b-C r e E R$ animals $(n=8)$ served as controls. Representative co-IF images for tdTom, tdTom/CK19, tdTom/Ki67, and tdTom/acetylated tubulin are shown that demonstrate reduced proliferative expansion and impaired biliary maturation of tdTom ${ }^{+}$DRCs after both NOTCH inactivation and/or WNT/ $\beta$-catenin activation (arrows indicate tdTom ${ }^{+}$DRCs that coexpress Ki67 or acetylated tubulin). In all models, DRCs failed to undergo lineage conversion to hepatocytes, as assessed by tdTom/HNF4 $\alpha$ co-IF analysis. (B) Quantification of co-IF data (mean value \pm SEM expressed in percentage; ${ }^{*} P<0.05,{ }^{* *} P<0.01,{ }^{* *} P<0.001[1$-way ANOVA followed by Tukey's posttest]). Scale bar: $100 \mu \mathrm{m}$ (first column); $20 \mu \mathrm{m}$ (second through fifth columns). 
long-term model (positive trend in short-term models), suggesting that, besides DRC-to-hepatocyte differentiation, more effective proliferative expansion of DR-derived hepatocytes may contribute to the small increase of tdTom ${ }^{+} / \mathrm{HNF} 4 \alpha^{+}$hepatocytes in the CDE diet models (Supplemental Figure 3B).

In all models, we found no evidence that lineage-marked tdTom ${ }^{+}$DRs had emerged from mesenchymal cells or expressed mesenchymal markers during the course of injury (Supplemental Figure 4). Remarkably, in long-term DDC treatment, we observed a growing number of $\mathrm{A6}^{+}$(and/or SOX9+) hepatocytes and cells of intermediate morphology (hepatobiliary cells), which were not found in the $M d r 2^{-/-}$or CDE models (Figure 3C and Supplemental Figure 5A). These cells surrounded typical A6 ${ }^{+} / \mathrm{CK} 19^{+}$DRs and could be interpreted to represent progenitors entering the hepatocyte lineage by light microscopy. However, we did not find DDC-induced $\mathrm{A}^{+} / \mathrm{HNF} 4 \alpha^{+}$hepatocytes or hepatobiliary cells that costained for tdTom (Figure 3C), clearly demonstrating their hepatocyte origin. These hepatocyte-derived $\mathrm{A}^{+} / \mathrm{HNF} 4 \alpha^{+}$cells may further maturate to $\mathrm{CK} 19^{+} / \mathrm{HNF} 4 \alpha^{-}$biliary cells and contribute to the $\mathrm{CK} 19^{+} \mathrm{DRs}$ after long-term DDC, as recently suggested by hepatocyte fate mapping $(6,8)$. However, the absolute contribution of hepatocyte-derived DRCs to DDC-induced CK19+ DRs was low, even after 8 months of DDC long-term treatment, as the proportion of $\mathrm{tdTom}^{+} / \mathrm{CK} 19^{+} \mathrm{DRs}$ decreased by only approximately 15\% (Supplemental Figure 5B).

Tamoxifen-inducible mouse models used for lineage tracing require careful characterization to ensure correct data interpretation (33). Different CreER strains may exhibit highly variable sensitivity to tamoxifen-induced reporter expression, depending on the strength of the Cre-driving promoter and the respective reporter strain used (34). Further, the possibility of tamoxifen-independent reporter expression as well as de novo recombination events caused potentially by tamoxifen that was not eliminated during injury must be considered. In our system, this Cre activation, perpetuated by remnant tamoxifen after the 7-day washout period, could lead to de novo labeling of HNF1 $\beta$-expressing ductal cells but also potentially mark injury-induced HNF1 $\beta$-expressing hepatocytes. This might lead to both underestimating the contribution of nonbiliary compartments to DRs and overestimating the number of duct-derived hepatocytes. However, we only found tamoxifen-dependent reporter expression in both homeostasis and injury (Supplemental Figure 6). Further, experiments measuring nuclear translocation of Cre, reporter expression over time, and HNF1 $\beta$ expression in nonductal compartments during injury (Supplemental Figure 7, A-F) as well as tamoxifen-spiking experiments (Supplemental Figure 7, G and H) oppose the concern of de novo reporter labeling of DRCs or hepatocytes after the 7-day washout as used throughout our study (refer to legend of Supplemental Figure 7 for a detailed description).

In vivo genetic modulation of NOTCH and/or WNT/ $\beta$-catenin signaling do not redirect $D R$ f from biliary differentiation toward the hepatocyte lineage. Our results challenge the view that the primary function of DRs is as progenitors to regenerate damaged hepatocytes and rather imply that the biliary fate of DRs in the postnatal mouse is firmly determined with limited plasticity over a broad range of acute and chronic injury modes. However, a highly reputed concept has been developed recently, implying that the opposing activities of NOTCH and WNT/ $\beta$-catenin signaling within DRCs are regulated by the DR-surrounding cellular niche and dictate the cellular differentiation of DRCs toward biliary cells or hepatocytes (10). This attractive and potentially clinically relevant hypothesis lacks confirmation from formal lineage tracing. Therefore, we combined our lineage-tracing approach with genetic inactivation of canonical NOTCH by deleting the essential NOTCH effector RBP-Jא and/or activation of the WNT/ $\beta$-catenin signaling pathway by expressing a nondegradable mutant of $\beta$-catenin specifically within the tdTom-labeled biliary compartment, hypothesizing that these genetic modifications would instruct DRCs toward the hepatocyte lineage.

We found the HNF1 $\beta^{+}$biliary compartment morphologically unaltered and no $\mathrm{tdTom}^{+} / \mathrm{HNF} 4 \alpha^{+}$hepatocytes when $\mathrm{R} 26^{\mathrm{Tom}}$ $R b p j^{i / f l} H n f 1 b$-CreER animals were analyzed up to 24 weeks after tamoxifen treatment, suggesting that canonical NOTCH is not absolutely required to maintain the biliary fate of bile ducts and ductules in normal homeostasis (data not shown). However, after DDC treatment, $R 26^{\text {Tom }} R b p j^{i l / f l} H n f 1 b$-CreER animals displayed a blunted tdTom ${ }^{+} \mathrm{DR}$, as assessed by morphometric analysis (Figure 4, A and B). Effective and specific genetic deletion of Rbpj within DDC-induced DRCs was confirmed by IHC analysis (Supplemental Figure 8A). Further, expression of the proliferation marker Ki67 and the cilia marker acetylated tubulin was reduced within RBP-J $\kappa$-deficient tdTom ${ }^{+}$DRCs (Figure 4, A and B), indicating that $\mathrm{NOTCH}$ is required for both effective expansion and biliary maturation of DRs. However, inactivation of NOTCH does not suffice to convert DDC-induced DRCs to hepatocytes, as tdTom ${ }^{+}$/ $\mathrm{HNF} 4 \alpha^{+}$hepatocytes were virtually absent (Figure 4, A and B).

Next, we analyzed mice expressing a nondegradable form of the WNT effector $\beta$-catenin within the tdTom-labeled ductal compartment ( $R 26^{\text {Tom }} \mathrm{Catnb}^{4 e x 3} \mathrm{Hnf1b}$-CreER mice). In these mice, tamoxifen treatment resulted in robust nuclear and cytoplasmic staining of $\beta$-catenin in DDC-induced DRCs $(75.0 \% \pm 1.3 \%)$ as compared with that in livers from equally treated $\mathrm{R}_{2} 6^{\text {Tom }} \mathrm{Hnflb}$ CreER animals $(3.38 \% \pm 0.58 \%$; Supplemental Figure $8 \mathrm{~B})$. Yet, this also failed to generate DRC-derived $\mathrm{tdTom}^{+} / \mathrm{HNF} 4 \alpha^{+}$hepatocytes (Figure 4, A and B). Even after simultaneous inactivation of Rbpj and expression of stabilized $C a t n b^{4 e x 3}$ in DDC-induced DRCs (R26 Tom Catnb ${ }^{\Delta e x 3} R b p f^{\text {Tl/fl }} H n f 1 b$-CreER mice), we failed to detect a significant number of $\mathrm{tdTom}^{+} / \mathrm{HNF} 4 \alpha^{+}$hepatocytes. These findings support the view that the biliary identity of postnatal DRs is rather static, with low potential to adopt a hepatocyte fate. Notably, $\beta$-catenin activation had similar inhibitory effects on DR expansion and biliary maturation as did NOTCH inhibition in the DDC model (Figure 4B), whereas IHC/immunofluorescence (IHC/IF) analysis of progenitor markers, such as CD133 or CD44, revealed similar expression in DRCs across all genotypes analyzed in Figure 4 (data not shown).

DRCs do not give rise to tumors in selected genetic or genotoxic mouse HCC models. The HCC cell type(s) of origin is unknown. Human and rodent HCCs may display progenitor cell gene signatures and/or contain LPC-like cells with cancer stem cell features (35). Consequently, adult bipotential LPCs have increasingly emerged as candidate cells for malignant transformations that give rise to HCC $(24,25)$. In support of this concept, p53-deficient or H-RAS/SV4OLT-transduced isolated DRCs can generate HCC 
A $R 26^{\mathrm{Tom}} \mathrm{Hnf1b}-\mathrm{CreER}$
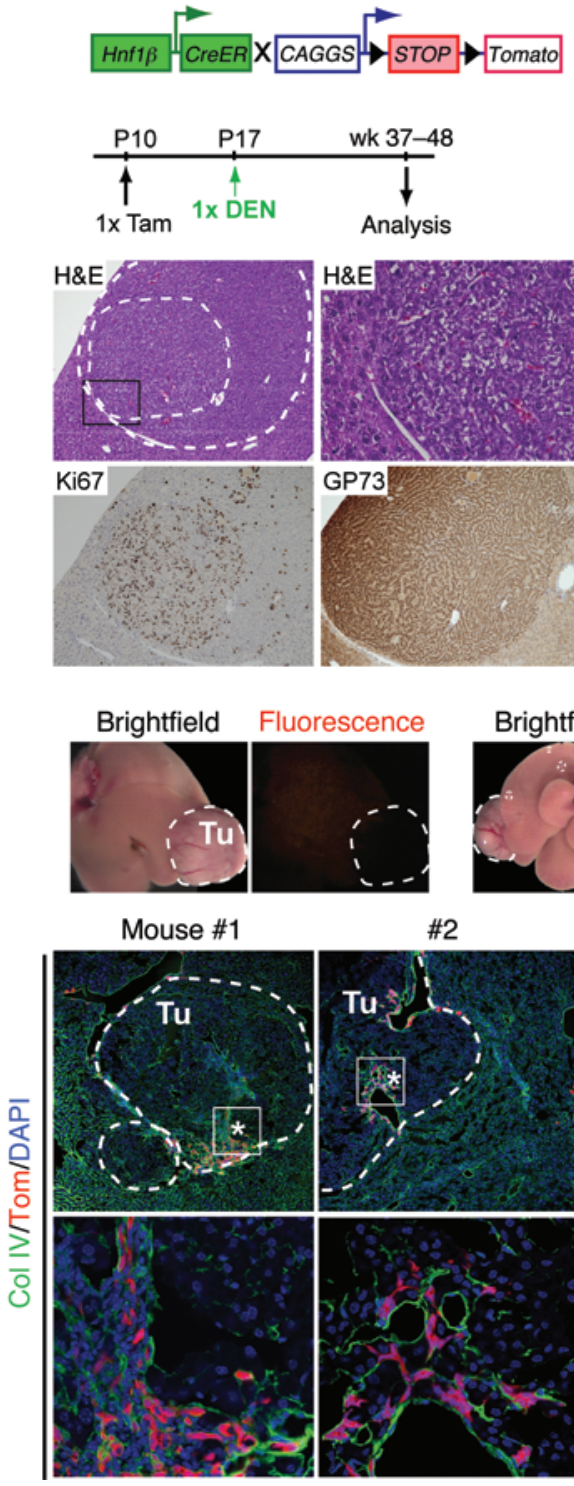

Mouse \#1

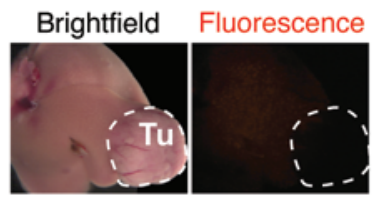

\#2

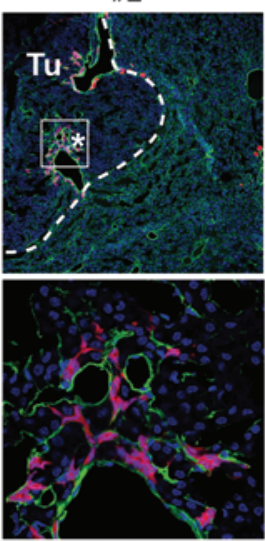

Brightfield Fluorescence

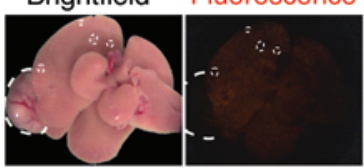

\#3

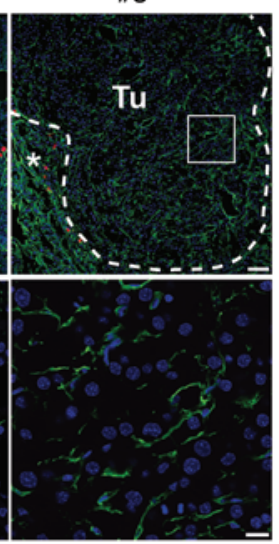

B
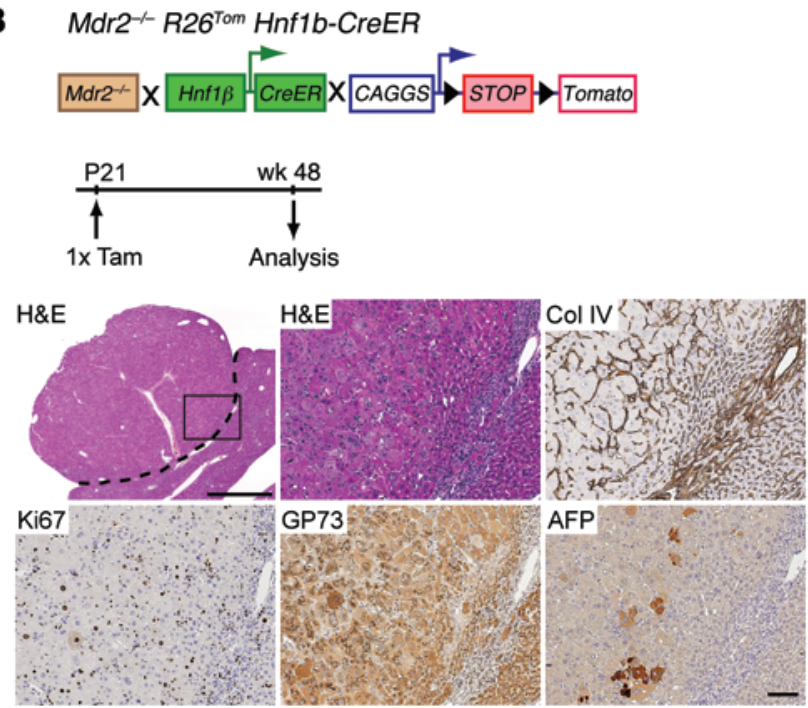

Mouse \#1

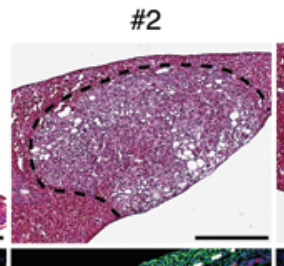

\#2
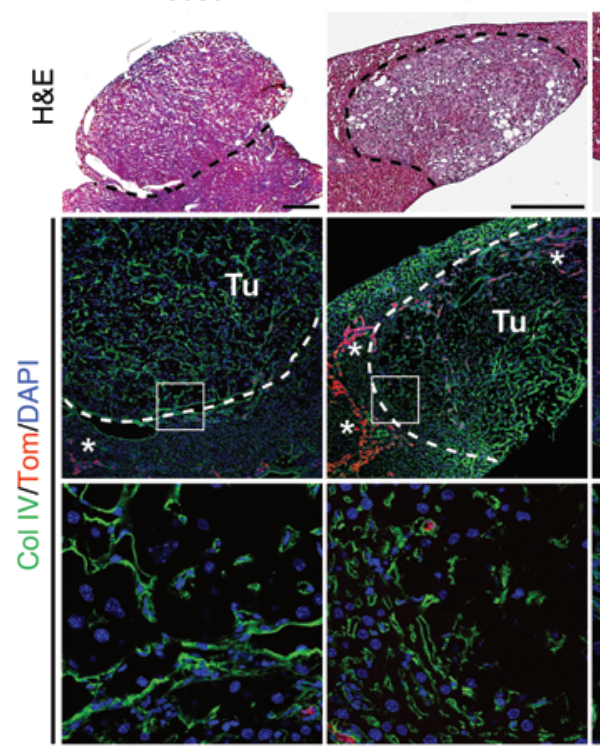

\#3
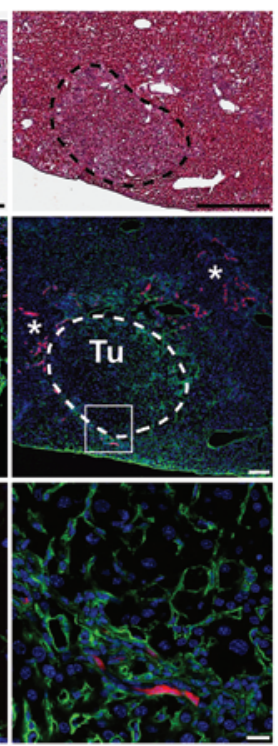

Figure 5. DRCs do not give rise to tumors in selected mouse HCC models. (A) R26 Tom Hnf1b-CreER animals (tamoxifen at P10) were injected with DEN (25 $\mathrm{mg} / \mathrm{kg} \mathrm{BW})$ and analyzed at 10 to 12 months after DEN treatment $(n=6)$. Representative IHC micrographs of serial sections obtained from one DEN-induced tumor nodule stained for collagen IV (Col IV), Ki67, GP73, or AFP. Representative macroscopic transmitted light and fluorescent whole-liver images from one DEN-treated animal show all nodular lesions (encircled by dashed lines) negative for tdTom. Co-IF analysis for tdTom and collagen IV revealed sporadic tdTom $^{+}$DRCs (asterisks) at the tumor margins and within the tumor nodules, but tumor cells never costained for tdTom (tumor nodules obtained from different animals are encircled by dashed lines; insets are shown at higher magnification below). (B) Likewise, characterization of tumors observed in Mdr2R26 Tom Hnf1b-CreER animals (tamoxifen at P21) was performed by IHC for collagen IV, Ki67, GP73, and AFP in 12-month-old animals; nodule formation was observed in 4 out of $n=6$ animals. Representative serial cryosections of tumor nodules processed from different mice for H\&E and co-IF for tdTom and collagen IV indicate tumor cells negative for tdTom (encircled by dashed lines). tdTom+ DRCs (asterisks) are typically observed outside tumor margins and rarely found within. Tu, tumor. Scale bar: 1,000 $\mu$ m (low-magnification H\&E, top panel, B); $500 \mu \mathrm{m}$ (H\&E mouse 1-3, B); $100 \mu \mathrm{m}$ (low-magnification H\&E, IHC, and top row IF, A; high-magnification H\&E, IHC, and top row IF, B); $20 \mu \mathrm{m}$ (high-magnification H\&E and bottom row IF, A; bottom row IF, B).

in xenotransplant models $(36,37)$. However, whether HCCs with or without progenitor features arise from transformed progenitors or rather from dedifferentiated/transformed hepatocytes, or both, is currently unknown (for review see ref. 25).

Although we could not identify a predominant role of biliary-derived DRCs in hepatocyte neogenesis after injury, few hepatocytes were DRC derived, indicating bilineage potential of at least some DRCs. Due to the high labeling efficacy in our lineage-tracing model, we hypothesized that we would detect tdTom $^{+}$HCC nodules if adult biliary-derived DRCs were indeed targets to malignant transformation in HCC models. Therefore, we analyzed $R 26^{\text {Tom }} \mathrm{Hnf1b}$-CreER animals treated with carcinogenic diethylnitrosamine (DEN) at P17 that had been pretreated with tamoxifen at P10; we confirmed these animals to be equally effective in tdTom labeling HNF1 $\beta^{+}$biliary cells (analysis at P17: $92.7 \% \pm 3.8 \%, n=3$ ). When analyzed at 10 to 12 months after DEN 
injection, livers of all $R 26^{\text {Tom }} \mathrm{Hnflb}$-CreER animals displayed tumor nodules (mean $6.0 \pm 2.8$ nodules, $n=6$ animals), showing typical features of HCC when characterized by histology and IHC markers, including collagen IV, Ki67, Golgi protein 73 (GP73), and alpha fetoprotein (AFP) (Figure 5A). However, macroscopically visible HCCs or microscopically identified tumors never expressed fluorescent tdTom when analyzed by fluorescence stereomicroscopy or confocal microscopy (Figure $5 \mathrm{~A}$ ). Next, we analyzed HNF1 $\beta$ lineage-labeled $M d r 2$-deficient ( $M d r 2^{-/}$R26 $6^{\text {Tom }}$ Hnflb-CreER) mice. $M d r 2^{-/-}$animals are widely used as a genetic model for spontaneous, inflammation-induced HCC development $(38,39)$, with extensive activation of progenitors that might undergo malignant transformation. Four of six animals analyzed at 12 months had developed tumor nodules (mean $4.0 \pm 3.1$ ). These tumors were highly proliferative and displayed broadening of liver cords, with loss of collagen IV, a coarse block pattern of perinuclear GP73, and frequent intermingling of $\mathrm{AFP}^{+}$hepatocytes (Figure 5B). However, as in DEN-treated livers, tumor cells in $\mathrm{Mdr2^{-- }} R 26^{\text {Tom }} \mathrm{Hnflb}$ CreER animals never expressed tdTom in coimmunofluorescence (co-IF) analysis (Figure 5B). These data clearly indicate that the neoplastic lesions in the DEN and $M d r 2^{-/-}$HCC models are not derived from DRCs but rather originate from hepatocytes.

\section{Discussion}

First, our results demonstrate that irrespective of the mode and duration of injury, CK19+ DRs arise almost exclusively from the adult biliary compartment. Second, DRCs or LPCs do not significantly contribute to hepatocyte regeneration in acute or long-term chronic liver injury. Third, there is no "streaming liver," in which hepatocytes are continuously replaced by cells originating from a progenitor cell niche in the biliary compartment or from zone 1 hepatocytes in a portal-to-central vein direction. Fourth, genetic modulation of the lineage-defining NOTCH and WNT/ $\beta$-catenin signaling pathways cannot release DRCs or LPCs from their biliary fate in vivo. Fifth, tumors in classical genetic or genotoxic mouse HCC models do not originate from DRCs.

DRs found in liver disease have been defined as cellular reactions of ductular phenotype but not necessarily of ductular (biliary) origin (3). Our results show that such DRs derive from the biliary compartment in short-term liver injury models, as the initial high fraction of reporter-labeled CK19+ biliary cells was maintained in CK19+ DRs. Earlier reports of hepatocytes as an additional source for DRCs (5) have recently been confirmed by hepatocyte lineage tracing in the setting of cholestatic liver injury $(6,8)$. This stepwise biliary conversion of adult hepatocytes can be induced by genetic NOTCH activation $(7,40)$ and relies on canonical NOTCH signaling upon injury (6). Using AAV-based specific labeling of the adult hepatocyte compartment, Yanger et al. found that up to $14 \%$ of $\mathrm{CK} 19^{+}$DRCs may arise from hepatocytes, with increasing duration of injury most pronounced in the DDC model (6). Although we did not set out to trace hepatocyte fates, the findings with our model of hepatocyte reprogramming to $\mathrm{CK} 19^{+}$biliary cells are compatible with our biliary fate-tracing model, in which we found increasing numbers of $\mathrm{A}^{+}$and/or SOX $9^{+}$hepatobiliary cells that never coexpressed the tdTom reporter in long-term DDC-induced injury models. Initial reporter labeling of CK19+ DRs (DDC diet for 2 weeks) was consistently reduced after long-term injury by about $15 \%$, further corroborating the conception that a minor portion of DRCs derive from hepatocytes. In contrast with our study and Yanger's study, Suzuki and colleagues proposed that the vast majority of all DDC-induced CK19+ DRs arise from the hepatocyte and not from the biliary compartment, even after short-term DDC treatment, when using the Alb-CreER strain (41) to specifically trace the hepatocyte compartment (9). To address these discrepancies, we used the identical Alb-CreER mouse strain (41) to analyze Cre activity in $R 26^{\text {Tom }}$ Alb-CreER animals. However, we found tdTom reporter activity not only in hepatocytes but also in approximately $40 \%$ of $\mathrm{CK} 19^{+}$small biliary ductules 1 and 7 days after low-dose tamoxifen (Supplemental Figure 9A), indicating that Cre expression in Alb-CreER animals lacks hepatocyte specificity. Consequently, $40 \%$ of DDC-induced CK19+ DRs around peripheral portal tracts costained for tdTom (Supplemental Figure 9B), which could erroneously be interpreted to arise from hepatocyte-to-biliary conversion.

The concept that adult DRs may harbor facultative liver stem cells to replace hepatocytes in case of severe injury has been deduced from rat models, in which the liver is forced to regenerate by $\mathrm{PH}$ after exposure to hepatotoxins such as acetylaminofluorene (AAF/PH model), which is thought to abrogate hepatocyte selfrenewal. In the course of such models, characteristic DRs, traditionally termed "oval cell response," appear along with hepatobiliary cells of transitional morphology, expressing both biliary and hepatocyte markers (42), suggesting that these DRs undergo hepatocyte differentiation. In vitro protocols that allow differentiation of isolated DRCs either to biliary cells or hepatocytes support the concept of bipotential DRCs/oval cells in the rat liver. Deduced from these rat studies, the theory of DRCs/oval cells being capable to serve as an SOS compartment for physiologically acting liver stem cells has been widely acknowledged as a basic principle of liver regeneration $(43,44)$; however, the lack of adequate lineage-labeling techniques in rats prohibits such definite conclusion (45).

Support for the theory of adult biliary-derived DRs having high bipotential capacity was provided by Furuyama et al., who performed formal lineage tracing of the biliary compartment using an inducible Sox9-CreER knock in mouse (18). They concluded that $\mathrm{SOX} 9^{+}$biliary cells give rise to a large fraction of new hepatocytes, not only in various liver injury models, but also in normal liver homeostasis, in which they provide a continuous hepatocyte supply (18), thereby reviving the controversial "streaming liver" hypothesis (32). Neogenesis of hepatocytes from the SOX9 compartment has also been reported after DDC diet using a different, transgenic Sox9-CreER mouse strain (14) identical to the one used in our study. However, biliary compartment specificity of adult Sox9-CreER mouse strains has been challenged $(4,20)$. In line with these reports, we found periportal reporter expression in $9 \%$ of hepatocytes. Furthermore, subsequent studies fate mapping the biliary compartment using different transgenic mouse models (Opn-CreER or Hnf1b-CreER) found no contribution of the biliary compartment or DRCs to homeostatic hepatocyte turnover and very limited contribution to hepatocyte neogenesis after shortterm liver injury $(19,21)$.

Although the quantity of DR-derived hepatocytes after shortterm injury was low in these mouse studies $(19,21)$, advocates for the theory of DRs acting as physiologic progenitors speculated 
that long-term injury might well lead to accumulation of a relevant number of DR-derived hepatocytes. In line with these reports, we found negligible (DDC diet, BDL, MDA, $M d r 2^{--}$, $80 \% \mathrm{PH}$, retrorsine/ $\mathrm{PH}$ ) or very low ( $\mathrm{CDE}$ diet) hepatocyte neogenesis from DRCs in the short-term injury and regeneration models. However, despite dramatic expansion of DRs in 3 different long-term models, we found virtually no (DDC diet, $M d r 2^{-/}$) or minimal (1.5\% in the CDE model) contribution of DRCs to hepatocyte origination.

In two recent articles, the hepatocyte-producing capacity of mouse biliary-derived DRCs was contested completely, even at low frequency $(22,23)$. This conclusion was derived from 2 to 3 weeks of short-term CDE diet in models using selective inducible Ck19CreER-or AAV8-Cre-mediated lineage labeling of the ductal ( $10 \%-$ $35 \%$ labeling) or hepatocyte ( $99 \%$ labeling) compartment, and no (23) or a negligible number $(<0.1 \%)$ (22) of DRC-derived hepatocytes were detected. Besides the possibility that a subset of DRCs may not be marked using the Ck19-CreER strain, experimental conditions, including start of the CDE diet in the animals at an older age, may also account for the failure to detect rare events of DRC-to-hepatocyte differentiation in these studies. In rodents, the magnitude of the DR in response to the CDE diet is highly age dependent (46), and lineage maturation of mouse biliary cells is concordant with a decline in their capacity to become hepatocytes in vitro after the neonatal period (47). At the same time, potential limitations of our model system must be considered. Ductal lineage labeling was highly effective and specific after a single tamoxifen dose. This specificity was maintained during homeostasis, and we found no evidence that remnant tamoxifen perpetuates de novo labeling of (injured) cells in our models (Supplemental Figure 7), suggesting that the small fraction of reporter-expressing hepatocytes observed in the CDE models is indeed duct derived. However, we did observe "ectopic" prelabeling of hepatocytes, despite this being an extremely rare event $(0.012 \%)$. As basally prelabeled hepatocytes, if detected at all, were exclusively found in zone 1, we cannot categorically rule out that the portion of labeled hepatocytes had increased in the CDE models as a result of selection of zone 1 hepatocytes after differential zonal liver damage of predominantly zone $2 / 3$ hepatocytes. Nevertheless, our conclusion that DRC-derived hepatocytes insignificantly contribute to short- and long-term injury repair remains intact.

Biliary compartment-derived DRs are highly heterogeneous in morphology. Yet, it is unknown whether different morphological phenotypes also reflect different cellular origins within the biliary compartment. Recent studies demonstrated that the transcriptional profiles of isolated DDC- and CDE-induced DRs differ (10, 21), and Boulter et al. have convincingly shown that the cellular DRsurrounding niche can influence the transcriptome of DRCs (10). Presupposing a common precursor of DDC- and CDE-induced bipotential DRs, the authors proposed that the balance of $\mathrm{NOTCH}$ and $\mathrm{WNT} / \beta$-catenin signaling determines the biliary $(\mathrm{NOTCH}-$ driven) or hepatocyte (WNT/ $\beta$-catenin-driven) fate of DRCs. To support this concept, the authors expressed a stabilized active $\beta$-catenin mutant specifically in $\mathrm{CK}_{1} 9^{+}$biliary cells and found nuclear $\beta$-catenin in periportal hepatocytes in the DDC model. Although a formal lineage-tracing approach was not implemented, the authors concluded that $\sim 10 \%$ of all hepatocytes had arisen from lineage conversion of primarily biliary-destined DRCs (10). However, when combining our lineage-tracing model with genetic modulation of
NOTCH and/or $\beta$-catenin specifically in DDC-induced DRCs, we found altered DR expansion but were unable to divert DRs from their biliary fate to become hepatocytes. This supports the concept that the biliary fate of DDC-induced DRs is firmly fixed and that NOTCH signaling and WNT/ $\beta$-catenin determine cellular cues for effective DR proliferation and morphogenesis $(7,12,48)$, rather than for DRC lineage allocation. Our findings that neoplastic lesions did not arise from the biliary HNF1 $\beta$ lineage in two distinct, commonly used HCC mouse models further fits in this overall picture of limited plastic capacity of postnatal biliary cells and highly suggests that hepatocytes are the primary cell type susceptive to malignant transformation in hepatocellular carcinogenesis.

Currently, to the best of our knowledge, there is no animal model that unequivocally proves the concept that biliary-derived DRCs or LPCs physiologically contribute significantly to hepatocyte neogenesis in adults, when lineage segregation of the hepatocyte and biliary compartments from hepatoblasts has matured. For zebrafish larvae, two highly recognized studies have convincingly shown that the perinatal biliary compartment can give rise to hepatocytes after severe liver damage $(49,50)$; however, although the authors also concluded that this holds true in the adult setting, inducible lineage tracing of biliary cells was not included in the adult zebrafish studies in either report, precluding the proof of such product-precursor relationship.

In all our models, hepatocyte replication was never abolished completely. Therefore, we cannot dogmatically rule out that experimental scenarios of liver failure (i.e., after complete and strictly hepatocyte-specific growth arrest not affecting the biliary compartment), in which DRCs may, in fact, rescue hepatocyte mass, as shown in transplantation studies, may exist. Whether such situations are of relevance for human disease and whether complete hepatocyte growth arrest will ultimately imply irrecoverable liver failure need to be addressed by future research and novel models. Further, the exceptionally high fraction of polyploid hepatocytes in mice may confer special resistance and self-renewal capacity to the hepatocyte compartment in this species (51). However, considering the widespread appearance of biliary-derived DRs in multiple mouse injury models, with no or minimal contribution to hepatocyte neogenesis in our study, it seems unlikely that such a highly conserved injury response has evolved to chiefly provide a progenitor pool for new hepatocytes. The question then arises of the main physiological function of DRs. One possible answer is that DRs provide an anatomical biliary network to preserve biliary drainage from the site of hepatic injury. Limited neogenesis of DR-derived hepatocytes and limited biliary conversion of hepatocytes might also help to restore the continuity between the hepatocyte canalicular system and biliary ductules (52), depending on the type of injury. Moreover, even without giving rise to hepatocytes directly, DRs (together with their obligatory surrounding mesenchymal and inflammatory niche) may well provide important paracrine signals that assist self-renewal of hepatocytes.

Although the hypothesis of biliary-derived DRCs serving as a physiologically significant cellular backup pool for hepatocytes in liver disease still awaits validation, the clonal and bipotential capacity of adult biliary-derived cells in vitro is beyond dispute (45). In this context, it has been recently demonstrated that human biliary cells obtained from biopsy can be expanded, 
cultivated long-term as organoids, and subsequently converted to functional hepatocytes in vitro and in vivo, which may offer enormous therapeutic potential (53). Nevertheless, in the current literature, there has been an inflationary use of the terms adult "liver stem cells" or "liver progenitor cells" for any biliary or progenitor marker-expressing DRCs, regardless of their ill-defined biological functions. Owing to the fact that, up to now, there is no definite evidence that DRCs function as physiologic bipotential progenitors in a quantitatively relevant way in adult liver disease, we discourage the broad use of these terms for DRCs until the explicit function of DRCs is firmly established for each setting of injury.

\section{Methods}

Animals and tamoxifen treatment. Tamoxifen-inducible Hnflb-CreER (54), Sox9-CreER (30), and Alb-CreER (41) mouse strains all have been described previously and were crossed with Ai14-R26 $6^{\text {lox-STOP-lox-tdTom/+ }}$ (referred to as $R 26^{\text {Tom }}$ ) reporter mice (26) (The Jackson Laboratory, stock 007914) to obtain R26 ${ }^{\text {Tom }} \mathrm{Hnf1b}$-CreER, R26 ${ }^{\text {Tom }}$ Sox9-CreER, and $R 26^{\text {Tom }}$ Alb-CreER mice that express the fluorescent dye tdTom upon Cre expression. For inducible inactivation of canonical NOTCH signaling, R2 $^{\text {Tom }} \mathrm{Hnf1b}$-CreER animals were further crossed with mice that carry floxed alleles for $R b p j$ (referred to as $R b p j^{j / f l}$ ) (55) to obtain $R 26^{\text {Tom }}$ $R b p j^{i l / f l} H n f 1 b$-CreER mice; analogue interbreedings were performed with $C a t n b^{\text {lox(ex3) }}$ animals (56) for conditional expression of a nondegradable active form of $\beta$-catenin ( $R 26^{\text {Tom }} \mathrm{Catnb}^{4 e x 3} \mathrm{Hnflb}$-CreER animals) or with multidrug resistance 2 ( $M d r 2$, also known as $A b c b 4)$ gene knockout ( $\left.M d r 2^{-/}\right)$mice (57) (The Jackson Laboratory, stock 002539) to obtain $M d r 2^{--}$R2 $6^{\text {Tom }}$ Hnflb-CreER animals. All mouse strains were maintained on a mixed background. For sequences of synthetic oligonucleotides used for genotyping refer to Supplemental Table 1.

To induce Cre activation in Hnflb-CreER and Sox9-CreER mice, tamoxifen (dissolved in corn oil) (Sigma-Aldrich) was injected once (i.p.) at $100 \mu \mathrm{g} / \mathrm{g}$ BW into 4 - to 5-week-old mice, unless otherwise specified in the figure legends. Cre recombinase was activated in AlbCreER mice with a single injection of low-dose tamoxifen at $100 \mathrm{ng} / \mathrm{g}$ BW. In all experimental models, tamoxifen was administered at least 7 days before different injury models were started.

Models for liver injury, regeneration, and carcinogenesis. For dietinduced liver injury, mice were fed a choline-deficient (MP Biomedicals), DL-ethionine-supplemented (0.165\% in $\mathrm{H}_{2} \mathrm{O}$, Sigma-Aldrich) diet (CDE diet) or a DDC diet (0.1\% DDC in standard diet, Altromin) ad libitum (19). BDL was performed in 8-week-old animals using inhalation anesthesia with isoflurane. After midline laparotomy, the common bile duct was separated from the hepatic artery and ligated twice. $\mathrm{PH}$ was performed in 7- to 8-week-old animals as described previously (27). For $2 / 3 \mathrm{PH}$, the left lobe, the left median lobe, and the right median lobe were ligated separately and resected. For $80 \% \mathrm{PH}$, the lower right lobe and one omental lobe were additionally removed. For inhibition of hepatocyte proliferation (28) before $2 / 3 \mathrm{PH}$, retrorsine (SigmaAldrich) was added to demineralized water at $10 \mathrm{mg} / \mathrm{ml}$ and titrated to $\mathrm{pH} 2.5$ with $35 \% \mathrm{HCl}$ diluted 1:10. The solution was neutralized to $\mathrm{pH}$ 7.0 with $1 \mathrm{M} \mathrm{NaOH}$, and $\mathrm{NaCl}$ was added to obtain a concentration of $0.15 \mathrm{M} \mathrm{NaCl}$. Animals were injected weekly for 2 weeks i.p. at $70 \mu \mathrm{g} / \mathrm{g}$ BW before PH was performed. Acute toxic biliary injury was induced by i.p. injection of 4,4'-methylene dianiline (MDA/DAPM) 3 times on days 0,3 , and 6 at $50 \mu \mathrm{g} / \mathrm{g} \mathrm{BW}$ (58). For this, MDA (Sigma-Aldrich) was dissolved in $100 \%$ ethanol and further diluted to $50 \mathrm{mg} / \mathrm{ml}$ in $35 \%$ ethanol at $42^{\circ} \mathrm{C}$. For hepatocarcinogenesis, male mice received a single injection (i.p.) of $25 \mathrm{mg} / \mathrm{kg} \mathrm{BW}$ of DEN (Sigma-Aldrich) at P17 (59). In all experiments, animals were monitored for BW, physical activity, grooming routines, levels of alertness, and other signs of stress using specific score sheets for each animal model as approved.

IF and IHC. For standard histological and IHC analysis, livers were removed, fixed in 4\% PFA for 20 hours, embedded in paraffin, and sectioned. Serial 3.5- $\mu \mathrm{m}$-thick sections were stained with $\mathrm{H} \& \mathrm{E}$ using standard protocols. IHC was performed with primary antibodies (Supplemental Table 2) followed by diaminobenzidine staining (Vector Laboratories) and counterstaining with hematoxylin. For IF analysis, livers were fixed in $4 \%$ PFA for 2 hours at $4^{\circ} \mathrm{C}$; washed in PBS; cryoprotected with increasing concentrations of $10 \%, 20 \%$, and $30 \%$ sucrose solutions prepared in PBS; and embedded in O.C.T. Tissue Tek. Serial 10- $\mu$ m-thick cryosections were stained with different primary antibodies (Supplemental Table 2), followed by fluorescent secondary antibodies (Supplemental Table 2) and nuclei costaining with DAPI or ToPro3 (pictures were taken with a Zeiss Axiowert 200M microscope or confocal Olympus FV10i microscope).

Image J software $(1.47 \mathrm{v})$ was used to determine the $\mathrm{tdTom}^{+}$area (\%). We analyzed 15 to 25 images at a $\times 100$ magnification from $\geq 3$ discontinuous liver sections obtained from 2 to 3 different liver lobes per mouse. Further quantitative analysis of IF data was performed by counting $\mathrm{tdTom}^{+}$coexpression markers $\left(\mathrm{CK} 19^{+}\right.$or $\left.\mathrm{HNF} 4 \alpha^{+}\right)$and all positive marker cells; data were expressed as percentages. For this, 15-25 images per mouse (at $\times 600$ magnification for CK19+ marker cells and $\times 100$ magnification for $\mathrm{HNF} 4 \alpha^{+}$marker cells) were taken from $\geq 3$ liver sections from 2 different lobes and quantified, unless otherwise specified in the figure legends.

Statistics. Data are presented as mean value \pm SEM. Sample size for each experiment is indicated in the figure legends. Statistical comparisons were made using either 1-way ANOVA followed by Tukey's post-test or unpaired, 2-tailed Student's $t$ test with GraphPad Prism software. A $P$ value of less than 0.05 was considered significant.

Study approval. Mice were handled and experiments were performed according to the protocols approved by Technische Universität München Institutional Animal Care and the veterinarian office of the State of Bavaria (Regierung von Oberbayern, approval ID: TVA 55.21-54-2531-55-07 and 55.2-1-54-2532-173-12) according to the National Institutes of Health Guide for the Care and Use of Laboratory Animals (8th ed. The National Academies Press. 2011.).

\section{Acknowledgments}

This work was supported by the German Research Foundation (DFG) (GE 2289/1-2 to F. Geisler and SI 1549/1-1 to J.T. Siveke) and by the German Cancer Consortium (DKTK) (to R.M. Schmid and J.T. Siveke). M. Ringelhan received a clinical leave stipend funded by the German Centre for Infection Research (DZIF). M. Sander was supported by an NIH/National Institute of Diabetes and Digestive and Kidney Diseases award (DK078803). A6 antibody was a gift from Valentina Factor. Alb-CreER animals were provided by Pierre Chambon. Catnb ${ }^{\text {lox(exz) }}$ animals were a gift from Makoto M. Taketo.

Address correspondence to: Fabian Geisler, 2nd Dept. of Internal Medicine, Klinikum rechts der Isar, Technische Universität München, Ismaninger Str. 22, 81675 München, Germany. Phone: 49.89.4140.5972; E-mail: fabian.geisler@lrz.tum.de. 
1. Gouw AS, Clouston AD, Theise ND. Ductular reactions in human liver: diversity at the interface. Hepatology. 2011;54(5):1853-1863.

2. Desmet VJ. Ductal plates in hepatic ductular reactions. Hypothesis and implications. I. Types of ductular reaction reconsidered. Virchows Arch. 2011;458(3):251-259.

3. Roskams TA, et al. Nomenclature of the finer branches of the biliary tree: canals, ductules, and ductular reactions in human livers. Hepatology. 2004;39(6):1739-1745.

4. Carpentier R, et al. Embryonic ductal plate cells give rise to cholangiocytes, periportal hepatocytes and adult liver progenitor cells. Gastroenterology. 2011;141(4):1432-1438.

5. Michalopoulos GK, Barua L, Bowen WC. Transdifferentiation of rat hepatocytes into biliary cells after bile duct ligation and toxic biliary injury. Hepatology. 2005;41(3):535-544.

6 . Yanger K, et al. Robust cellular reprogramming occurs spontaneously during liver regeneration. Genes Dev. 2013;27(7):719-724.

7. Jeliazkova $P$, et al. Canonical Notch2 signaling determines biliary cell fates of embryonic hepatoblasts and adult hepatocytes independent of Hes1. Hepatology. 2013;57(6):2469-2479.

8. Tarlow BD, et al. Bipotential adult liver progenitors are derived from chronically injured mature hepatocytes. Cell Stem Cell. 2014;15(5):605-618.

9. Sekiya S, Suzuki A. Hepatocytes, rather than cholangiocytes, can be the major source of primitive ductules in the chronically injured mouse liver. Am J Pathol. 2014;184(5):1468-1478.

10. Boulter L, et al. Macrophage-derived Wnt opposes Notch signaling to specify hepatic progenitor cell fate in chronic liver disease. Nat Med. 2012;18(4):572-579.

11. Spee B, et al. Characterisation of the liver progenitor cell niche in liver diseases: potential involvement of Wnt and Notch signalling. Gut. 2010;59(2):247-257.

12. Geisler F, Strazzabosco M. Emerging roles of Notch signaling in liver disease. Hepatology. 2015;61(1):382-392.

13. Suzuki A, et al. Flow cytometric isolation and clonal identification of self-renewing bipotent hepatic progenitor cells in adult mouse liver. Hepatology. 2008;48(6):1964-1978.

14. Dorrell C, et al. Prospective isolation of a bipotential clonogenic liver progenitor cell in adult mice. Genes Dev. 2011;25(11):1193-1203.

15. Huch M, et al. In vitro expansion of single Lgr5+ liver stem cells induced by Wnt-driven regeneration. Nature. 2013;494(7436):247-250.

16. Okabe M, et al. Potential hepatic stem cells reside in EpCAM+ cells of normal and injured mouse liver. Development. 2009;136(11):1951-1960.

17. Malato Y, et al. Fate tracing of mature hepatocytes in mouse liver homeostasis and regeneration. J Clin Invest. 2011;121(12):4850-4860.

18. Furuyama K, et al. Continuous cell supply from a Sox9-expressing progenitor zone in adult liver, exocrine pancreas and intestine. Nat Genet. 2010;43(1):34-41.

19. Espanol-Suner R, et al. Liver progenitor cells yield functional hepatocytes in response to chronic liver injury in mice. Gastroenterology.
2012;143(6):1564-1575.

20. Tarlow BD, Finegold MJ, Grompe M. Clonal tracing of Sox $9(+)$ liver progenitors in mouse oval cell injury. Hepatology. 2014;60(1):278-289.

21. Rodrigo-Torres D, et al. The biliary epithelium gives rise to liver progenitor cells. Hepatology. 2014;60(4):1367-1377.

22. Schaub JR, Malato Y, Gormond C, Willenbring H. Evidence against a stem cell origin of new hepatocytes in a common mouse model of chronic liver injury. Cell Rep. 2014;8(4):933-939.

23. Yanger K, et al. Adult hepatocytes are generated by self-duplication rather than stem cell differentiation. Cell Stem Cell. 2014;15(3):340-349.

24. Rountree CB, Mishra L, Willenbring H. Stem cells in liver diseases and cancer: recent advances on the path to new therapies. Hepatology. 2012;55(1):298-306.

25. Yamashita T, Wang XW. Cancer stem cells in the development of liver cancer. J Clin Invest. 2013;123(5):1911-1918.

26. Madisen L, et al. A robust and high-throughput Cre reporting and characterization system for the whole mouse brain. Nat Neurosci. 2010; 13(1):133-140.

27. Ringelhan M, Schmid RM, Geisler F. The NF-kappaB subunit RelA/p65 is dispensable for successful liver regeneration after partial hepatectomy in mice. PLoS One. 2012;7(10):e46469.

28. Kamiya A, Kakinuma S, Yamazaki Y, Nakauchi H. Enrichment and clonal culture of progenitor cells during mouse postnatal liver development in mice. Gastroenterology. 2009;137(3):1114-1126.

29. Iverson SV, Comstock KM, Kundert JA, Schmidt EE. Contributions of new hepatocyte lineages to liver growth, maintenance, and regeneration in mice. Hepatology. 2011;54(2):655-663.

30. Kopp JL, et al. Sox9+ ductal cells are multipotent progenitors throughout development but do not produce new endocrine cells in the normal or injured adult pancreas. Development. 2011;138(4):653-665

31. Zong Y, et al. Notch signaling controls liver development by regulating biliary differentiation. Development. 2009;136(10):1727-1739.

32. Zajicek G, Oren R, Weinreb M Jr. The streaming liver. Liver. 1985;5(6):293-300.

33. Lemaigre FP. Determining the fate of hepatic cells by lineage tracing: Facts and pitfalls [published online ahead of print December 12, 2014] Hepatology. doi:10.1002/hep.27659.

34. Liu J, Willet SG, Bankaitis ED, Xu Y, Wright $\mathrm{CV}, \mathrm{Gu}$ G. Non-parallel recombination limits Cre-LoxP-based reporters as precise indicators of conditional genetic manipulation. Genesis. 2013;51(6):436-442.

35. Lee JS, et al. A novel prognostic subtype of human hepatocellular carcinoma derived from hepatic progenitor cells. Nat Med. 2006;12(4):410-416.

36. Dumble ML, Croager EJ, Yeoh GC, Quail EA. Generation and characterization of p53 null transformed hepatic progenitor cells: oval cells give rise to hepatocellular carcinoma. Carcinogenesis. 2002;23(3):435-445

37. Holczbauer A, et al. Modeling pathogenesis of primary liver cancer in lineage-specific mouse cell types. Gastroenterology. 2013;145(1):221-231.

38. Pikarsky E, et al. NF-kB functions as a tumour promoter in inflammation-associated cancer. Nature. 2004;431(7007):461-466.

39. Pusterla T, et al. Receptor for advanced glycation endproducts (RAGE) is a key regulator of oval cell activation and inflammation-associated liver carcinogenesis in mice. Hepatology. 2013;58(1):363-373.

40. Fan B, et al. Cholangiocarcinomas can originate from hepatocytes in mice. J Clin Invest. 2012;122(8):2911-2915.

41. Schuler M, Dierich A, Chambon P, Metzger D. Efficient temporally controlled targeted somatic mutagenesis in hepatocytes of the mouse. Genesis. 2004;39(3):167-172.

42. Evarts RP, Nagy P, Marsden E, Thorgeirsson SS. A precursor-product relationship exists between oval cells and hepatocytes in rat liver. Carcinogenesis. 1987;8(11):1737-1740.

43. Duncan AW, Dorrell C, Grompe M. Stem cells and liver regeneration. Gastroenterology. 2009;137(2):466-481.

44. Tanaka M, Itoh T, Tanimizu N, Miyajima A. Liver stem/progenitor cells: their characteristics and regulatory mechanisms. J Biochem. 2011;149(3):231-239.

45. Miyajima A, Tanaka M, Itoh T. Stem/progenitor cells in liver development, homeostasis, regeneration, and reprogramming. Cell Stem Cell. 2014;14(5):561-574.

46. Guest I, Ilic Z, Sell S. Age dependence of oval cell responses and bile duct carcinomas in male fischer 344 rats fed a cyclic choline-deficient, ethionine-supplemented diet. Hepatology. 2010;52(5):1750-1757.

47. Tanimizu N, Nakamura Y, Ichinohe N, Mizuguchi T, Hirata K, Mitaka T. Hepatic biliary epithelial cells acquire epithelial integrity but lose plasticity to differentiate into hepatocytes in vitro during development. J Cell Sci. 2013; 126(pt 22):5239-5246.

48. Fiorotto R, et al. Notch signaling regulates tubular morphogenesis during repair from biliary damage in mice. J Hepatol. 2013;59(1):124-130.

49. He J, Lu H, Zou Q, Luo L. Regeneration of liver after extreme hepatocyte loss occurs mainly via biliary transdifferentiation in zebrafish. Gastroenterology. 2014;146(3):789-800.e8.

50. Choi TY, Ninov N, Stainier DY, Shin D. Extensive conversion of hepatic biliary epithelial cells to hepatocytes after near total loss of hepatocytes in zebrafish. Gastroenterology. 2014;146(3):776-788.

51. Duncan AW. Aneuploidy, polyploidy and ploidy reversal in the liver. Semin Cell Dev Biol. 2013;24(4):347-356.

52. Theise ND, Dolle L, Kuwahara R. Low hepatocyte repopulation from stem cells: a matter of hepatobiliary linkage not massive production. Gastroenterology. 2013;145(1):253-254.

53. Huch M, et al. Long-term culture of genomestable bipotent stem cells from adult human liver. Cell. 2015;160(1-2):299-212.

54. Solar M, et al. Pancreatic exocrine duct cells give rise to insulin-producing $\beta$ cells during embryogenesis but not after birth. Dev Cell. 2009;17(6):849-860.

55. Nakhai $\mathrm{H}$, et al. Conditional ablation of Notch signaling in pancreatic development. Develop- 
ment. 2008;135(16):2757-2765.

56. Harada $\mathrm{N}$, et al. Intestinal polyposis in mice with a dominant stable mutation of the $\beta$-catenin gene. EMBO J. 1999;18(21):5931-5942.

57. Smit JJ, et al. Homozygous disruption of the murine mdr2 P-glycoprotein gene leads to a complete absence of phospholipid from bile and to liver disease. Cell. 1993;75(3):451-462.

58. Kanz MF, Kaphalia L, Kaphalia BS, Romagnoli E, Ansari GA. Methylene dianiline: acute toxicity and effects on biliary function. Toxicol Appl Pharmacol.1992;117(1):88-97.

59. Sarma DS, Rao PM, Rajalakshmi S. Liver tumour promotion by chemicals: models and mechanisms. Cancer Surv. 1986;5(4):781-798. 